ARTICLE

\title{
A membrane-bound ankyrin repeat protein confers race-specific leaf rust disease resistance in wheat
}

Markus C. Kolodziej (1) 1, Jyoti Singla1, Javier Sánchez-Martín (10 1, Helen Zbinden', Hana Šimková (1) 2, Miroslava Karafiátová (10) 2, Jaroslav Doležel (10 2 , Julien Gronnier (D) 1, Manuel Poretti (1) 1, Gaétan Glauser ${ }^{3}$,

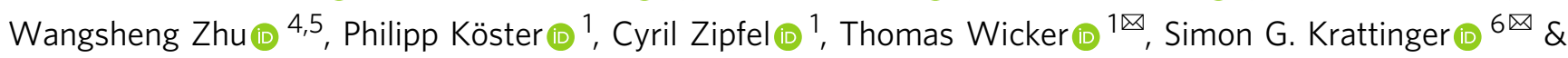
Beat Keller (1) ${ }^{1 \times}$

Plasma membrane-associated and intracellular proteins and protein complexes play a pivotal role in pathogen recognition and disease resistance signaling in plants and animals. The two predominant protein families perceiving plant pathogens are receptor-like kinases and nucleotide binding-leucine-rich repeat receptors (NLR), which often confer race-specific resistance. Leaf rust is one of the most prevalent and most devastating wheat diseases. Here, we clone the race-specific leaf rust resistance gene Lr14a from hexaploid wheat. The cloning of Lr14a is aided by the recently published genome assembly of ArinaLrFor, an Lr14a-containing wheat line. Lr14a encodes a membrane-localized protein containing twelve ankyrin (ANK) repeats and structural similarities to $\mathrm{Ca}^{2+}$-permeable non-selective cation channels. Transcriptome analyses reveal an induction of genes associated with calcium ion binding in the presence of Lr14a. Haplotype analyses indicate that Lr14a-containing chromosome segments were introgressed multiple times into the bread wheat gene pool, but we find no variation in the Lr14a coding sequence itself. Our work demonstrates the involvement of an ANK-transmembrane (TM)-like type of gene family in race-specific disease resistance in wheat. This forms the basis to explore ANK-TM-like genes in disease resistance breeding.

\footnotetext{
${ }^{1}$ University of Zurich, Department of Plant and Microbial Biology, Zollikerstrasse 107, 8008 Zurich, Switzerland. ${ }^{2}$ Institute of Experimental Botany of the Czech Academy of Sciences, Centre of the Region Hana for Biotechnological and Agricultural Research, Šlechtitelů 31, 77900 Olomouc, Czech Republic. ${ }^{3}$ Neuchâtel Platform of Analytical Chemistry, Université de Neuchâtel, Avenue de Bellevaux 51, 2000 Neuchâtel, Switzerland. ${ }^{4}$ Department of Molecular Biology, Max Planck Institute for Developmental Biology, 72076 Tübingen, Germany. ${ }^{5}$ College of Plant Protection, China Agricultural University, 100193 Beijing, China. ${ }^{6}$ King Abdullah University of Science and Technology (KAUST), Biological and Environmental Science and Engineering Division (BESE), Thuwal 23955-6900, Kingdom of Saudi Arabia. ${ }^{凶}$ email: wicker@botinst.uzh.ch; simon.krattinger@kaust.edu.sa; bkeller@botinst.uzh.ch
} 
eaf rust, caused by the fungal pathogen Puccinia triticina, is one of the most damaging wheat diseases ${ }^{1}$. Long-distance dispersal of $P$. triticina spores by wind facilitates the rapid spread of newly evolved leaf rust pathogen races $^{1}$. Genetic resistance within the wheat gene pool is the most sustainable way to control leaf rust ${ }^{1}$. Some wheat cultivars are characterized by exceptional levels of leaf rust resistance that continues to be effective in the field even after decades. Such durable and broadspectrum resistance is often the result of multiple, additively acting quantitative disease resistance genes. For instance, the Swiss winter wheat cultivar Forno (released in the 1980s) continues to show near immunity to all tested $P$. triticina races. The durable leaf rust resistance in Forno is controlled by several genes with additive effect ${ }^{2}$, including the adult plant resistance genes $L r 34^{3}$ and $L r 75^{4}$, and a race-specific all-stage resistance locus on chromosome arm 7BL that was postulated to be $\operatorname{Lr} 14 a^{2,4}$. Of these three genes, only $\operatorname{Lr} 14 a$ confers seedling resistance at ambient temperatures. A genetic interaction of $\operatorname{Lr} 14 a$ with $L r 75^{2}$ and a characteristic mesothetic seedling resistance phenotype with both fully developed leaf rust uredia and hypersensitive flecks occurring on the same leaf ${ }^{5}$ were observed. Earlier work on $\operatorname{Lr} 14 a$ has shown a strong dependence on environmental and genetic factors $^{5-8}$. These unique properties of phenotypic and genetic interactions of a major seedling disease resistance gene make Lr14a an interesting target for molecular functional analyses (Supplementary Note 1).

Here, we use two Forno-derived backcross lines, ArinaLrFor 4 ( $L r 75+L r 14 a$ in the genetic background of the susceptible Swiss winter wheat cultivar Arina) and ArinaLr14a, to isolate Lr14a. It encodes LR14A, an ankyrin repeat containing protein located in the plasma membrane and possibly acting as a non-selective, $\mathrm{Ca}^{2+}$-permeable cation channel to mediate resistance.

\section{Results and discussion}

Lr14a confers leaf rust resistance in ArinaLrFor. Both backcross lines and ThatcherLr14a ( $L r 14 a$ from the Canadian wheat cultivar Selkirk introgressed into the susceptible background of Thatcher) ${ }^{7}$ showed an Lr14a-characteristic mesothetic resistance response ${ }^{5}$ at the seedling stage (Fig. 1a). $F_{1}$ plants from a cross between Arina and ArinaLr14a were resistant, indicating that the Lr14a-mediated resistance is dominant (Supplementary Fig. 1a). Eleven tested $P$. triticina isolates produced identical avirulence/ virulence formulae on ArinaLrFor, ArinaLr14a, and ThatcherLr14a, corroborating that the gene on chromosome 7BL of ArinaLrFor is Lr14a (Supplementary Table 1). Adult ArinaLrFor and ArinaLr14a plants showed higher resistance compared to the susceptible parent Arina in the field (Supplementary Fig. 1b, c).

EMS mutants and VIGS identify and validate $\operatorname{Lr14a}$. To clone Lr14a, we identified seven seedling-susceptible mutant lines from two independent ArinaLrFor-derived ethyl methanesulfonate (EMS) mutant populations (Fig. 1b). Chromosome 7B of three of these mutants was isolated by flow cytometry and sequenced by short-read sequencing. Reads were aligned against the recently published chromosome-scale genome assembly of ArinaLrFor ${ }^{9}$. To identify candidate genes, the ArinaLrFor assembly was fragmented in silico and subjected to the MutChromSeq pipeline $e^{10}$. This resulted in the identification of a single gene-containing contig, in which all three mutant lines showed independent EMSinduced single nucleotide polymorphisms (SNPs) (G/C to A/T) compared to the wild-type in the predicted coding sequence (Fig. 1c). Amplification and sequencing revealed additional nonsynonymous SNPs in the four remaining mutants (Fig. 1c). To functionally validate the candidate gene, we performed virusinduced gene silencing (VIGS) by targeting two gene-specific sequences (Fig. 1c). Silencing of Lr14a in ArinaLrFor plants resulted in increased susceptibility apparent by increased numbers of leaf rust uredia and decreased numbers of hypersensitive flecks (Fig. 1d, e).

Lr14a encodes an ankyrin-transmembrane protein. The $\operatorname{Lr} 14 a$ coding sequence is $2340 \mathrm{bp}$ long, consists of three exons, and translates into a predicted 779 amino acid protein with an $\mathrm{N}$ terminal domain, containing 12 ankyrin (ANK) repeats, followed by six predicted transmembrane (TM) helices (Fig. 1c, Supplementary Fig. 2a-d). ANK repeat proteins have been described in archaea, bacteria, viruses, and eukaryots ${ }^{11}$. The ANK repeat domain consists of up to 30 repeats of a 33-amino acid motif, which forms two $a$-helices. ANK repeat domain-containing proteins either have the ANK repeat domain alone or in combination with various other domains, including TM domains, calmodulin binding domains, zinc finger domains, $\mathrm{K}^{+}$channel domains, and many more ${ }^{12}$. Several ANK proteins in plants play important roles in plant immunity, development, and growth, and they are typically involved in protein-protein interactions $^{12,13}$. No signal peptide was predicted in the $\mathrm{N}$ terminus of the LR14A protein and no alternative splicing was found. Two of the EMS mutants had SNPs in the sequence encoding the predicted TM domain, resulting in an amino acid substitution and in a premature stop codon, respectively. The remaining five mutants carry SNPs, resulting in amino acid substitutions in the predicted $\alpha$-helices that form the structural backbone of the ANK repeats. An identical amino acid substitution (L362F) was recovered from the two independent EMS populations. (Fig. 1c, Supplementary Fig. 2e).

Lr14a-specific diagnostic marker. An $\operatorname{Lr} 14 a$-specific presence/ absence marker, targeting the first exon of the gene (Supplementary Fig. 3a) was genetically mapped to the Lr14a region on chromosome arm 7BL (Supplementary Fig. 3b, Supplementary Note 1) in a bi-parental mapping population and showed complete co-segregation with the Lr14a-mediated rust resistance (Supplementary Data 1). The marker also amplified in the wheat lines Hope, Kalyansona, and Selkirk, which have been described as donor lines of $\operatorname{Lr} 14 a$ in wheat breeding 5 . In summary, our results from the mutagenesis experiment (Fig. 1b), genetic mapping (Supplementary Fig. 3b), and VIGS (Fig. 1d,e) demonstrate that Lr14a encodes an ANK-TM protein.

Lr14a phenotype is genotype and environment dependent. Within the bi-parental mapping population, Lr14a-containing lines showed a broad range of mesothetic resistance responses with varying proportions of hypersensitive flecks and leaf rust uredia. Differences in the magnitude of the Lr14a-mediated resistance response were also observed in various $\operatorname{Lr} 14 a$-containing wheat cultivars (Supplementary Fig. 3c). This is possibly the result of genetic modifiers, which have previously been described for $\operatorname{Lr} 14 a^{5,7,8}$ (Supplementary Note 1). The strong variability is reminiscent of quantitatively acting disease resistance genes ${ }^{14}$. Furthermore, the Lr14a gene action has been described as temperature sensitive ${ }^{6}$. Inoculation experiments at different temperatures were in agreement with previous reports ${ }^{12}$ and $L r 14 a$ was more effective at lower temperatures (Supplementary Fig. 4a).

Race-specific induction of $\mathbf{L r 1 4 a}$ expression. Lr14a expression was very low or undetectable in uninfected plants but increased two days post-inoculation (dpi) with the avirulent $P$. triticina isolate 96209. Expression peaked around four dpi, and then decreased again (Fig. 2a). In the susceptible EMS mutants, $\operatorname{Lr} 14 a$ 

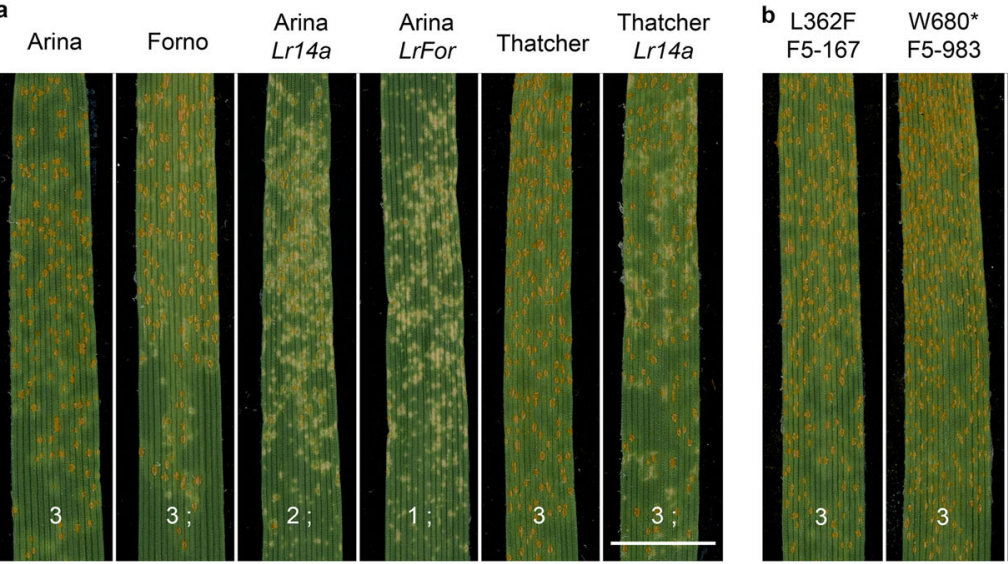

H178Y L362F

F10-7

G509R

P292L

G768S
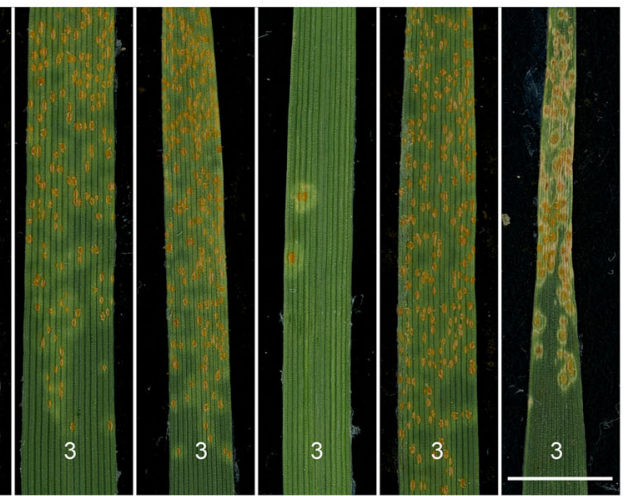

$\mathbf{c}$

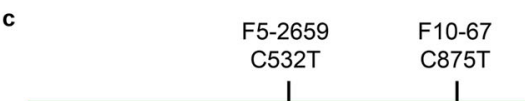

F5-167
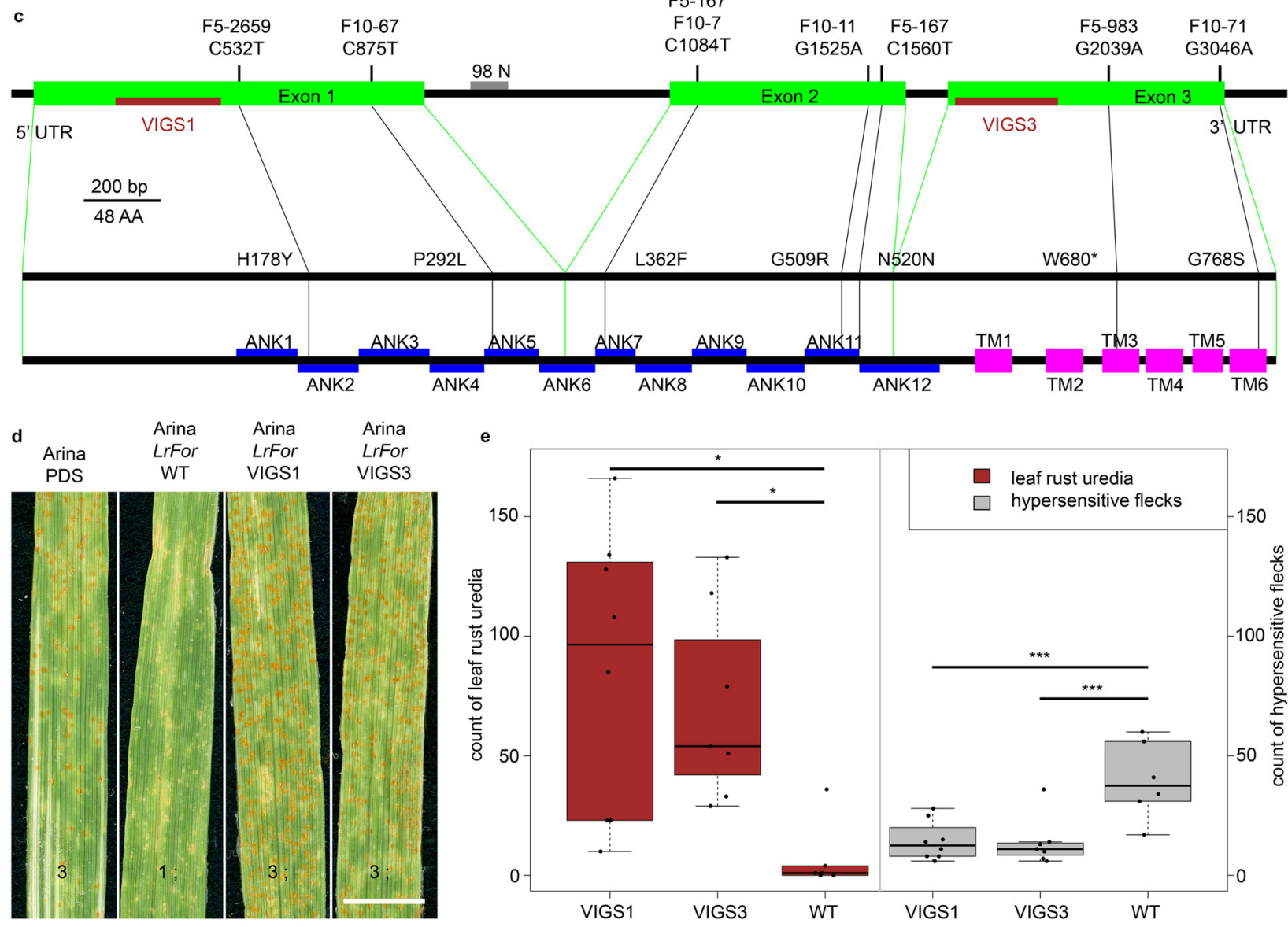

Fig. 1 Lr14a encodes an ANK-TM protein. a Seedling infections of wheat genotypes Arina, Forno, ArinaLr14a, ArinaLrFor, Thatcher, and ThatcherLr14a. b Seedling infections of ArinaLrFor-derived EMS mutants. Scale bars $=0.5 \mathrm{~cm}$. c Gene and protein structures of $L r 14 a$. $L r 14 a$ consists of three exons ( $g r e e n$ ).

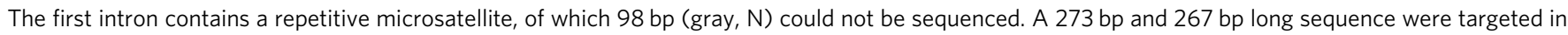
exons 1 and 3, respectively (brown) for virus-induced gene silencing (VIGS). The first four non-synonymous mutations are located in the N-terminal

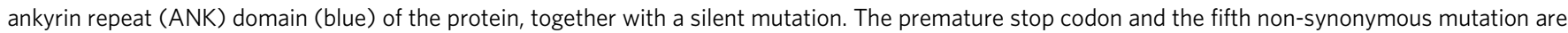

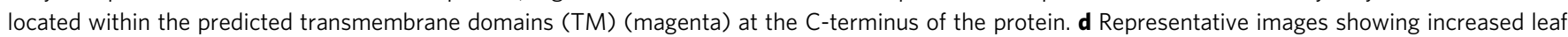
rust susceptibility after silencing the Lr14a candidate gene through VIGS. PDS = phytoene desaturase control, WT= barley stripe mosaic virus without silencing construct. Scale bar $=0.7 \mathrm{~cm}$. e Quantification of leaf rust symptoms. The numbers of leaf rust uredia (brown) and hypersensitive flecks (gray)

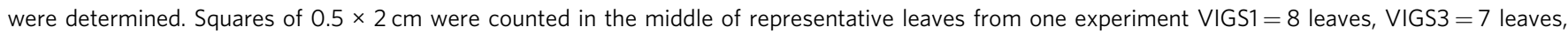
$\mathrm{WT}=6$ leaves. Statistics: for both datasets, a Levene test was performed, followed by ANOVA and a Shapiro-Wilk test for leaf rust uredia and Tukey-HSD for hypersensitive flecks. For boxplots: minimum value $=$ lower whisker, maximum value $=$ upper whisker, median $=$ middle value of box, lower quartile $=$

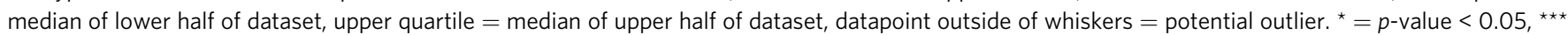
$=p$-value $<0.001$. Leaf rust infection types in (a), (b), and (d) are as follows: $1=$ small uredia with necrosis, $2=$ small to medium sized uredia with necrosis or chlorosis, $3=$ medium sized uredia with or without chlorosis, ; = hypersensitive flecks. Source data underlying (a), (b), (d), and (e) are provided as a Source Data file. 


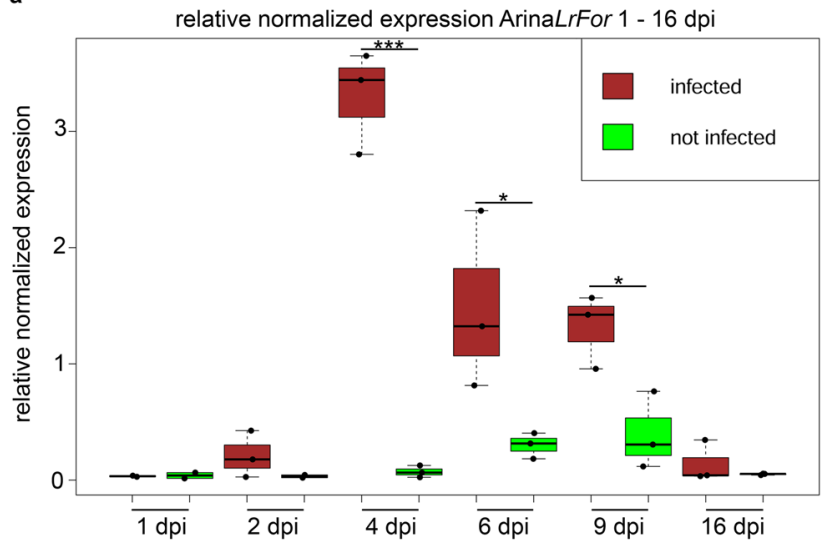

b
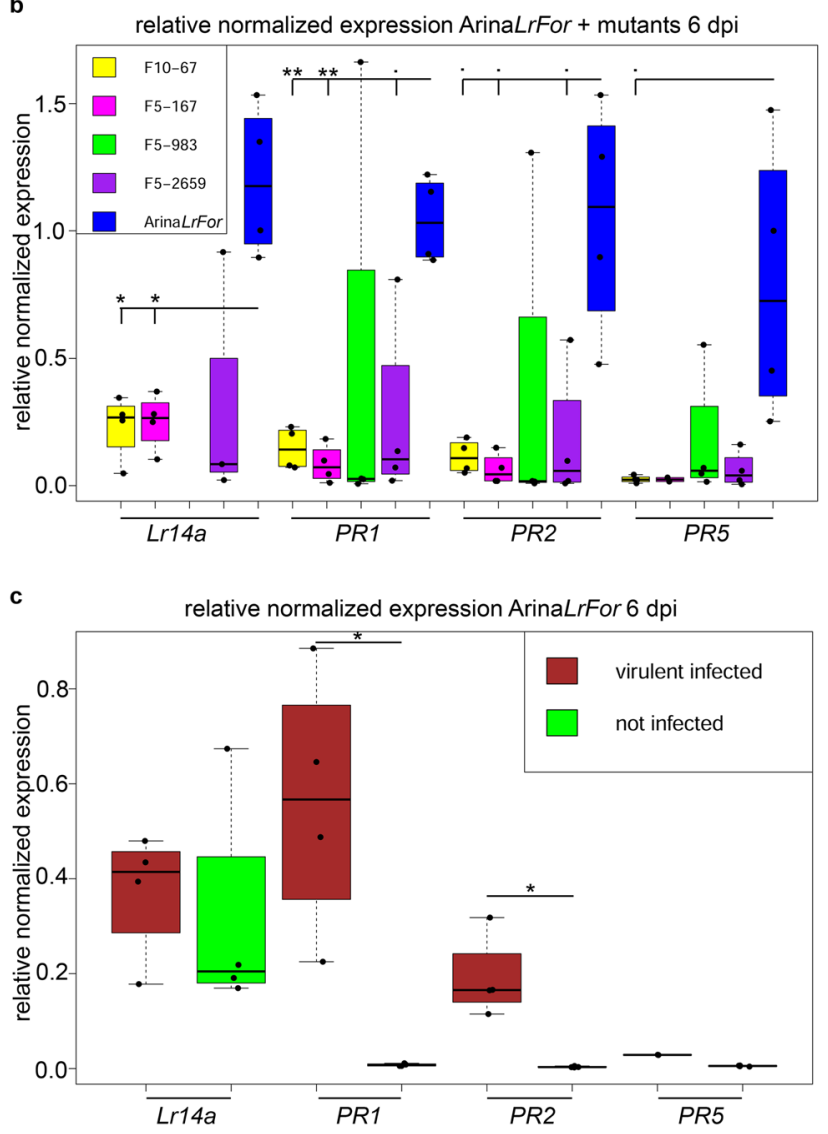

Fig. 2 Relative normalized expression of $\operatorname{Lr14a}$ and pathogenesis-related marker genes in different conditions. a, Lr14a expression in ArinaLrFor with and without infection (brown/green) with the avirulent Puccinia triticina isolate 96209. $n=3$. Statistics: Shapiro-Wilk test for each dataset was followed by Ftest for each timepoint comparison. 16 dpi was analyzed with an unpaired two-sided Wilcox test ( $p$-value $=0.7$ ). 1, 2, 4, 6, 9 dpi were analyzed with a one-sided t-test. $p$-values $=0.5861,0.161,0.0001153,0.02835,0.01289$, respectively. $\mathbf{b}$ Lr14a expression in ArinaLrFor was higher than expression in Lr14a EMS mutants (green one below qPCR detection limit), while pathogenesis-related (PR) marker expression in ArinaLrFor (blue) was higher than in the Lr14a EMS mutant lines (yellow, magenta, green, violet), 6 dpi with avirulent $P$. triticina isolate 96209. $n=4$. Statistics: Datasets of different gene expressions were analyzed by a Levene test, followed by a Welch-ANOVA one-way test for $L r 14 a, P R 1$, and PR2 and a Kruskal-Wallis test for PR5. c Lr14a and $P R$ marker expression of ArinaLrFor, 6 dpi with virulent $P$. triticina isolate 95037 (brown) and not infected (green). Lr14a transcript levels showed no significant differences between non-infected leaves and after inoculation with a virulent isolate. Two $P R$ genes were activated after infection with the virulent pathogen, while PR5 showed no detectable induction under the same conditions. $n=4$. Statistics: Shapiro-Wilk tests for each dataset were followed by F-test for each gene expression comparison. Wilcox test, twosided was used for $L r 14 a$ ( $p$-value $=0.6857)$, Welch test, two-sided was used for PR1 ( $p$-value $=0.02815)$ and PR2 ( $p$-value $=0.02377), P R 5$ was excluded from statistics because the infected condition has only two datapoints. For boxplots in (a), (b), (c): minimum value = lower whisker, maximum value $=$ upper whisker, median $=$ middle value of box, lower quartile $=$ median of lower half of dataset, upper quartile = median of upper half of dataset, datapoint outside of whiskers $=$ potential outlier. $\cdot=p$-value $<0.1,{ }^{\star}=p$-value $<0.05,{ }^{\star \star}=p$-value $<0.01,{ }^{\star \star \star}=p$-value $<0.001$. Source data are provided as a Source Data file.

showed different resistance responses, probably as a result of genetic modifiers $5,7,8$ (Supplementary Fig. 3c). The other 72 cultivars had a null-allele for the marker, indicating the absence of the gene. In all 27 wheat lines that were positive for the $L r 14 a$-derived marker, the Lr14a coding sequences were identical to the one found in ArinaLrFor. Two previously described alleles of $\operatorname{Lr} 14 a$, named $\operatorname{Lr} 14 b^{7}$ and $L r 14 c^{16}$ were investigated as well. The $L r 14 a$-derived marker failed to produce an amplification product in ThatcherLr14b. On the other hand, the marker amplified a product in the $\mathrm{Lr} 14 \mathrm{c}$ containing durum wheat line Creso. Amplification of the full-length coding sequence from Creso revealed $100 \%$ sequence identity to $L r 14 a$. We therefore concluded that $L r 14 b$ is not a true allele of $L r 14 a$ and that a modifier of resistance resulted in the misinterpretation of an $\mathrm{Lr} 14 \mathrm{c}$ allele (Supplementary Note 1). We extended our marker analysis to spelt (Triticum aestivum ssp. spelta), tetraploid wheat, and wild wheat relatives aiming to identify Lr14a diversity. Seven spelt lines (Supplementary Note 2) and 41 tetraploid Turkish durum cultivars and landraces, amongst them Hevidi (WW198) and Menceki (WW200) (Supplementary Fig. 3f), were positive for the Lr14a-specific marker (Supplementary Fig. 3a, Supplementary Data 2). The coding sequences of $L r 14 a$ were identical in all marker-positive spelt and durum wheat genotypes, suggesting a high degree of sequence conservation across different wheat subspecies. $\operatorname{Lr} 14 a$-containing spelt lines showed no resistance response when infected with the avirulent $P$. triticina isolate 96209 (Supplementary Fig. 3d). However, crosses between three of these spelt lines and the susceptible bread wheat cultivar Arina produced $\mathrm{F}_{1}$ plants with stronger seedling resistance (including mesothetic responses) than either parent after inoculation with $P$. triticina isolate 96209 (Supplementary Fig. 3e), providing evidence for the presence of $\operatorname{Lr14a}$ modifiers $5,7,8$ (Supplementary Note 2).

To further study the origin of $\operatorname{Lr} 14 a$, we analyzed collections comprising of 266 wild emmer wheat (Triticum turgidum ssp. diccocoides) accessions ${ }^{17}$ from Israel, Lebanon, and Turkey
Lr14a is highly conserved in the gene pool of grasses. We used the Lr14a-specific marker to assess the distribution of Lr14a in a global bread wheat collection (Supplementary Data 2). Out of 99 tested cultivars, 27 contained Lr14a. The Lr14a-positive lines 
a

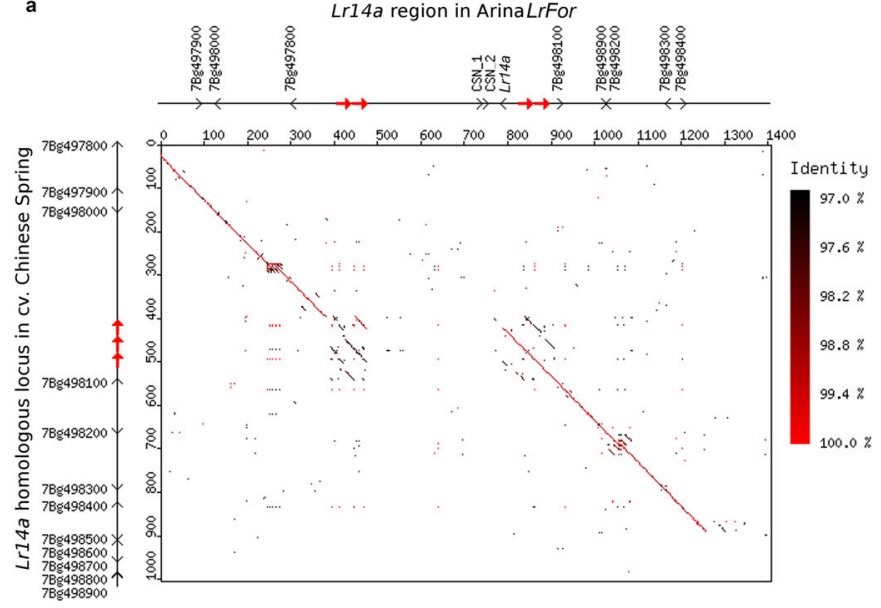

Loss of Lr14a through unequal recombination

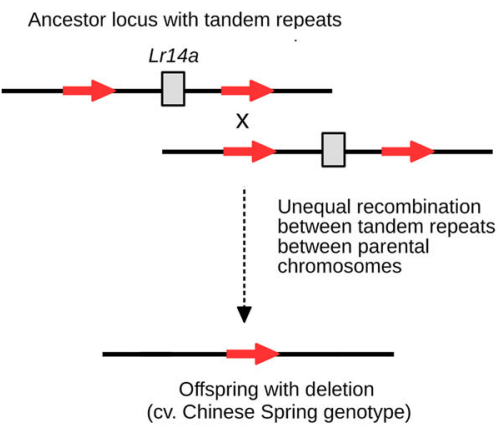

Fig. 3 Sequence comparison of the Lr14a locus in wheat cultivars ArinaLrFor and Chinese Spring. a Dotplot alignment of the Lr14a locus from ArinaLrFor (horizontal) and Chinese Spring (vertical). Sequence identity is color coded according to the scale at the right. Sequences are annotated with genes depicted as arrow heads to indicate transcriptional orientation and tandem repeats are depicted as red arrows. $\mathbf{b}$ Model for tandem repeats flanking the region containing Lr14a and their function as templates for unequal crossing-over that led to the deletion of the Lr14a gene in the Chinese Spring haplotype.

(Supplementary Data 2). The Lr14a-derived marker amplified in only eight wild emmer accessions collected near the Turkish cities of Gaziantep and Siverek close to the Turkish-Syrian border. Again, the Lr14a coding sequence was identical with $L r 14 a$ from ArinaLrFor in all the eight wild emmer accessions. These results indicate that $L r 14 a$ is rare in the wild emmer gene pool and only present in accessions from a particular geographic region. The absence of sequence diversity in wild emmer wheat, cultivated durum wheat landraces and hexaploid wheat suggests a low selection pressure in recent evolution which might possibly point to an indirect interaction of LR14A and the corresponding avirulence gene product from the leaf rust pathogen.

Lr14a's origin in the hexaploid bread wheat gene pool was traced back to a cross made in 1916 in South Dakota between the tetraploid, cultivated Yaroslav emmer and the bread wheat cultivar Marquis $^{5,18}$, which resulted in the famous bread wheat cultivar Hope. Yaroslav emmer and Hope were both positive for the Lr14agene-specific marker, showed a mesothetic resistance phenotype (Supplementary Fig. 3a, c), and their Lr14a coding sequences were identical to the sequence in ArinaLrFor. The origin of $L r 14 a$ in spelt is most likely different from the Yaroslav introgression because spelt and bread wheat separated thousands of years ago.

Multiple independent origins of Lr14a in the hexaploid wheat gene pool were also supported by analyzing the Lr14a locus in multiple recently published wheat genomes ${ }^{9}$. We found $\operatorname{Lr} 14 a$ in the genomes of bread wheat cultivar Lancer and spelt accession PI190962 ${ }^{9}$. Analyses of $\sim 60 \mathrm{~kb}$ of flanking regions indicated that these represent different haplotypes, which diverged from the ArinaLrFor haplotype approximately 44,000 and 10,000 years ago, respectively (Supplementary Note 3 ). Additionally, Chinese Spring contained an Lr14a-like haplotype, but lacked a $\sim 315 \mathrm{~kb}$ region containing $\operatorname{Lr} 14 a$, probably as a result of a deletion caused by unequal crossing over between large $(10-50 \mathrm{~kb})$ tandem repeats flanking the segment (Fig. 3, Supplementary Fig. 5, Supplementary Note 3, Supplementary Table 2).

The ANK-TM protein LR14A might act as $\mathrm{Ca}^{2+}$ channel. The ANK-TM gene family contains 15 members in maize, 37 members in rice, and 40 members in the model plant Arabidopsis thaliana ${ }^{13}$. One of the ANK-TM genes in Arabidopsis is Accelerated Cell Death 6 (ACD6) (AT4G14400), which has the same domain composition and shows $37 \%$ similarity to LR14A at the protein level (Supplementary Fig. 6a, b). LR14A protein homologs were described to be involved in quantitative disease resistance and stress response in Arabidopsis ${ }^{19-21}$ and most recently in maize 22 . Although all these proteins have the same ANK-TM domain structure, they share only a little sequence similarity with LR14A (Supplementary Fig. 6a, b). Phylogenetic analysis showed that the predicted LR14A protein is a member of a clade that has no counterpart in Arabidopsis (Supplementary Fig. 6c). Even within wheat, LR14A is unique and its closest homologs in ArinaLrFor and Chinese Spring only have a 75\% amino acid similarity. The closest Chinese Spring homolog is located on chromosome arm 5BL in a cluster with two other ANK-TM genes. Together with the low frequency and absence of polymorphisms in the Lr14a gene in tetraploid wild emmer wheat, this indicates a single introgression event in this species. However, the presence of sequence differences surrounding the gene suggests that Lr14a may have been subsequently introgressed multiple times into the hexaploid wheat gene pool.

Functional analyses of plant ANK-TM genes have mainly focused on ACD6, a key regulator of fitness tradeoffs between vegetative growth and general pathogen defense $\mathrm{e}^{23}$. The gain of function mutant acd6-1 has been reported to confer broadspectrum disease resistance. In contrast to the broad spectrum resistance of the autoactive acd6-1 with associated severe negative growth effects in Arabidopsis, $\operatorname{Lr} 14 a$ has a race-specific action and no $\operatorname{Lr} 14 a$-associated negative effects on plant vigor were found by measuring adult plant height and seedling above-ground weight (Supplementary Fig. $4 \mathrm{~b}-\mathrm{d}$ ). This is possibly the result of the absence of $\operatorname{Lr} 14 \mathrm{a}$ expression in the absence of avirulent leaf rust pathogen races (Fig. 2a, c). Located in the plasma membrane ${ }^{24}$, ACD6 forms large complexes with other proteins in the membrane ${ }^{25}$ and shows interactions with pattern recognition receptors like BAK1 and CERK $1{ }^{26}$. Overexpression of $\mathrm{N}$-terminal enhanced green fluorescence protein (eGFP) tagged LR14A in Nicotiana benthamiana epidermal cells revealed a plasma membrane localization of LR14A (Fig. 4). ACD6 expression is regulated by salicylic acid (SA) levels, and ACD6 regulates SA accumulation and signaling, particularly in the acd6-1 dominant gain-of-function mutant, which shows enhanced resistance to pathogens, amongst others due to elevated levels of SA $24,25,27$. We found no difference in salicylic acid (SA) levels between $P$. triticina inoculated ArinaLrFor plants and the EMS-induced mutants (Supplementary Fig. 1d), suggesting that LR14A might 


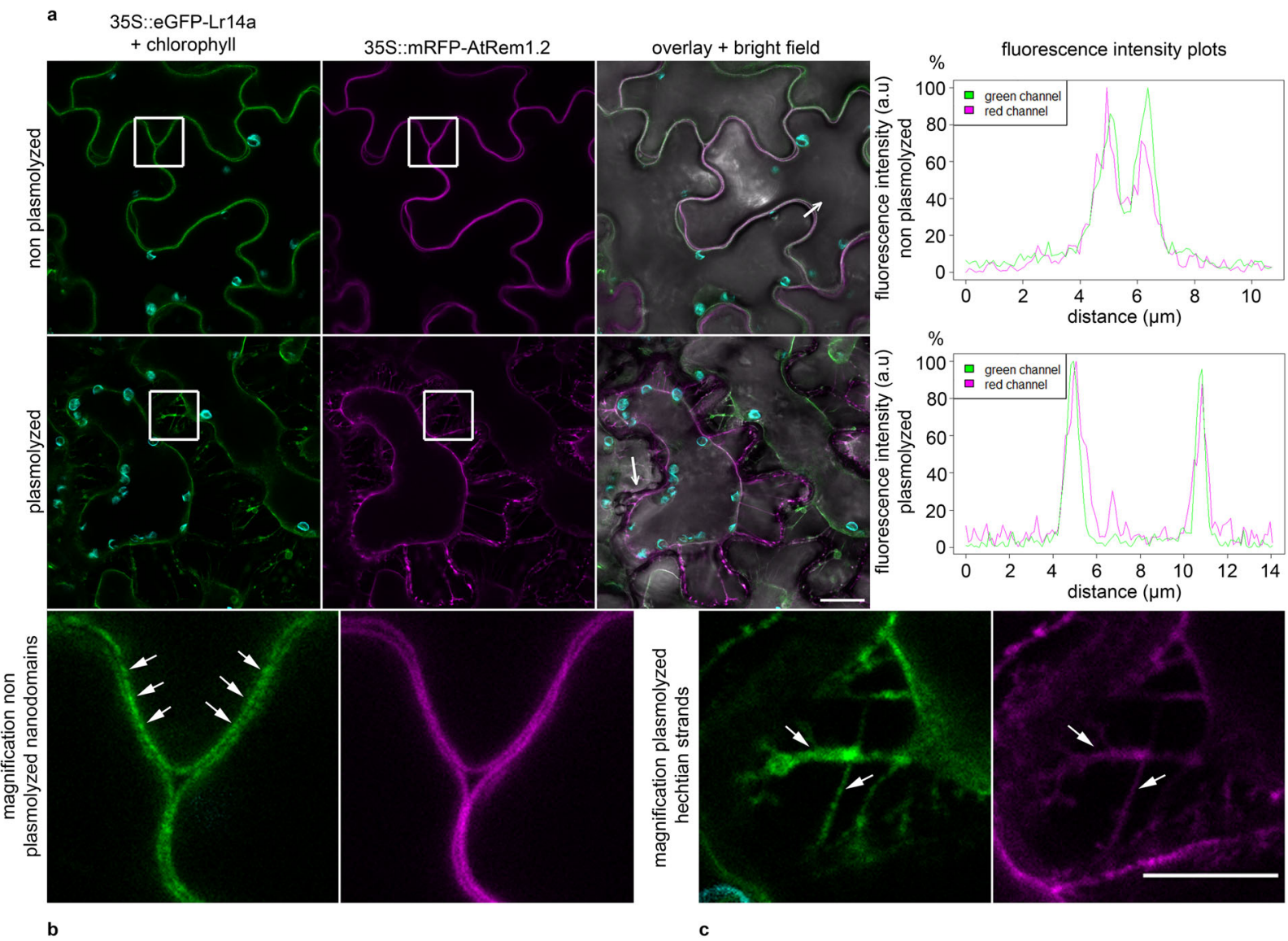

Fig. 4 LR14A localized to the plasma membrane. Nicotiana benthamiana leaves transiently transformed with Agrobacterium tumefaciens carrying an overexpression construct with enhanced green fluorescence protein (eGFP) tagged LR14A and monomeric red fluorescence protein mRFP tagged AtREM1.2, a plasma membrane (PM) marker63. Green pictures show the eGFP fluorescence as well as chlorophyll (turquoise). Magenta pictures show mRFP fluorescence. All fluorescence channels are overlaid in a bright field (BF) picture together with turquoise chloroplast autofluorescence. Nonplasmolyzed lower leaf surface cells showed co-localization of both proteins in the plasma membrane. The LR14A protein accumulated in nanodomains visible by different intensity of spots on the PM (see also magnification). Plasmolyzed cells of lower leaf surface cells showed the localization of the LR14A protein on the Hechtian strands (also see magnification). Hechtian strands are connections between PM and cell wall and an indication for a PM localization of the LR14A protein ${ }^{77}$. Fluorescence intensity (a. $\mathrm{u}=$ arbitrary unit) plots show co-localization of fluorescence signal (fluorescence intensity is expressed in \% of the maximum of fluorescence intensity of each channel) of eGFP and mRFP channel on not plasmolyzed PM and Hechtian strands over a $11 \mu \mathrm{m}$ or $14 \mu \mathrm{m}$ distance, respectively. Scale bar $=20 \mu \mathrm{m}$, for magnified images $10 \mu \mathrm{m}$. Source data are provided as a Source Data file.

act differently from ACD6. Interestingly, we identified a strong structural similarity between LR14A and calcium channels, including the transient receptor potential cation channel protein TRPA1 $^{28}$ (Supplementary Table 3 ). These results indicate that LR14A might function through an unknown resistance mechanism, possibly acting as a non-selective, $\mathrm{Ca}^{2+}$-permeable cation channel. To get further insight into the possible function of $L r 14 a$, we performed an RNAseq experiment in Thatcher and ThatcherLr14a. ThatcherLr14a infected with an avirulent leaf rust pathogen isolate showed 7,986 differentially expressed genes compared to Thatcher $8 \mathrm{dpi}$ and there was a significant differential expression for 160 genes associated with "calcium ion binding" (Fig. 5a, b, Supplementary Data 3,4,5). This reaction only occurred in ThatcherLr14a infected with an avirulent $P$. triticina isolate. Inoculation of Thatcher $L r 14 a$ with the virulent $P$. triticina isolate 95037 and mock inoculations resulted in a very low number of differentially expressed genes (65 and 193, respectively) and there were only 2 and 1 differentially expressed genes, respectively, associated with calcium ion binding (Fig. 5b). To further corroborate Lr14a's possible role in calcium fluxes, we transiently infiltrated $\operatorname{Lr} 14 a$ into $N$. benthamiana leaves and analyzed the differentially expressed genes $27 \mathrm{~h}$ post-infiltration
(Supplementary Fig. 7). Similar to wheat, we observed a significant enrichment for 26 genes associated with "calcium ion binding" in $N$. benthamiana leaves infiltrated with $\operatorname{Lr} 14 a$ compared to empty vector controls (Supplementary Data 6,7,8). Five of these genes were related to the A. thaliana homologous protein At5g02490 encoding a HEAT SHOCK COGNATE PROTEIN, 70-2 (HSC70-2), which has been described as a development and stress regulator ${ }^{29}$. Another 11 up-regulated genes are homologs of different CALMODULIN LIKE proteins (CMLs), which are calcium-sensing proteins involved in intracellular signaling ${ }^{30}$. Calcium is a known secondary messenger in plants involved in diverse signaling processes, including hormone regulation, abiotic, and biotic stress response ${ }^{31,32}$. The gene expression analysis suggests that $\operatorname{Lr} 14 a$, which is activated in wheat specifically after infection by an avirulent $P$. triticina isolate, is inducing genes involved in calcium signaling. The observation that infiltration of $\operatorname{Lr} 14 a$ alone results in similar changes of expression patterns in $N$. benthamiana further indicates that expression of $L r 14 a$ is sufficient for the induction of the observed changes in gene expression.

In $N$. benthamiana leaves, we observed a specific, Lr14adependent phenotype reminiscent to the previously described 
a
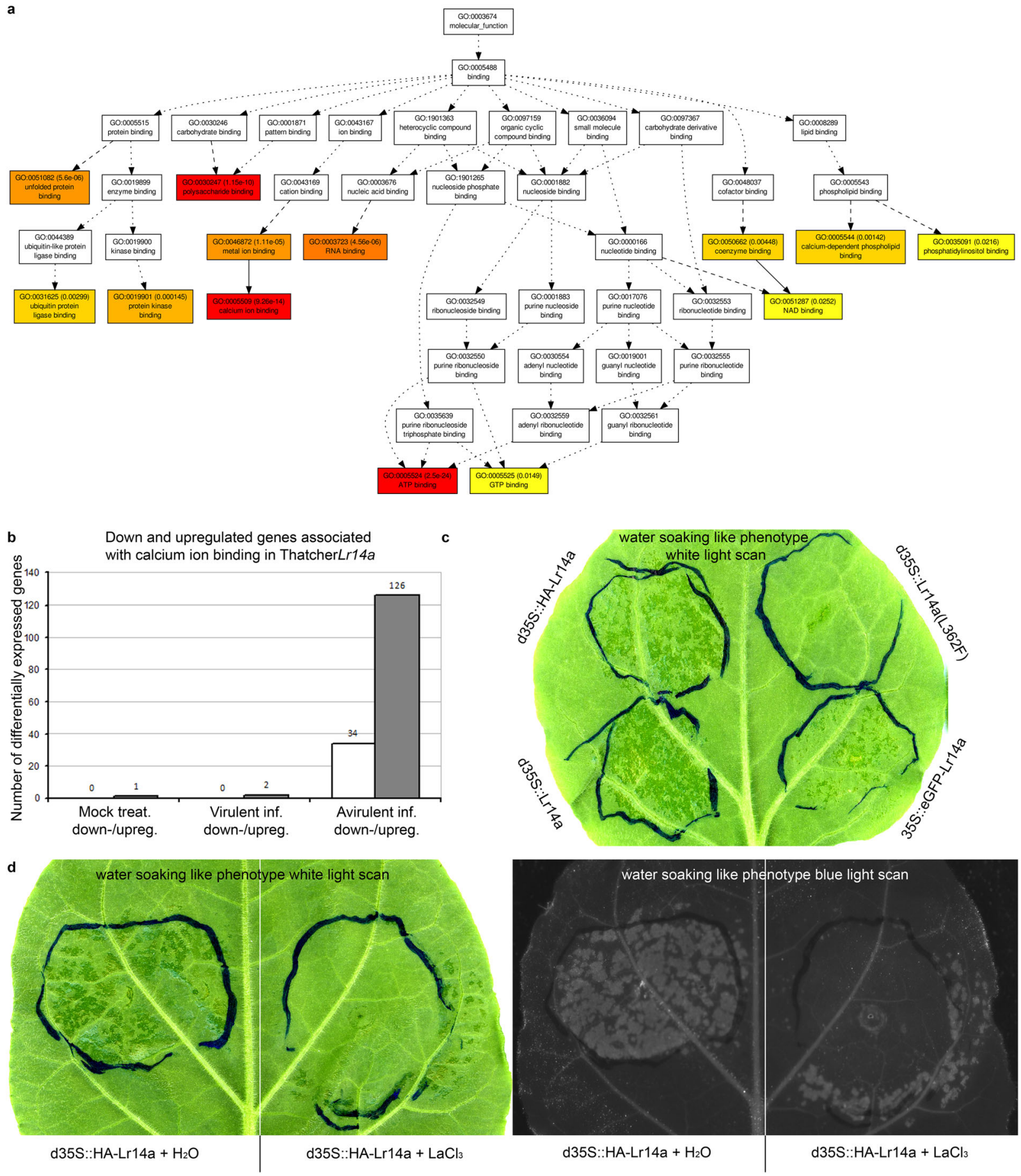

Fig. 5 LR14A induces calcium ion binding associated genes in wheat and Nicotiana benthamiana. a Gene ontology (GO) network graph showing that Lr14a causes induction of calcium ion binding associated genes 8 days post-inoculation (dpi) in the wheat line ThatcherLr14a compared to Thatcher after inoculation with an avirulent $P$. triticina isolate. b Number of differentially expressed genes that are associated with the GO term "calcium ion binding" in ThatcherLr14a compared to Thatcher after mock treatment and inoculation with a virulent and avirulent $P$. triticina isolate, respectively. c $N$. benthamiana leaves transiently transformed with Lr14a coding sequences representing wild-type (WT) Lr14a coding sequence, an EMS loss-of-function mutant (L362F) Lr14a coding sequence, an N-terminal enhanced green fluorescence protein (eGFP) tagged Lr14a coding sequence version, and an N-terminal human influenza hemagglutinin (HA) tagged Lr14a coding sequence. The functional Lr14a versions induced a water-soaking ${ }^{33}$-like phenotype on the lower leaf surface, $3 \mathrm{dpi}$. This phenotype was weaker in the eGFP tagged version, possibly due to steric hindrance of the LR14A function or differences in the expression associated with different vector systems. The LR14A loss of function mutant (L362F) lost its ability to cause the water-soaking-like phenotype in N. benthamiana. $\mathbf{d}$ The water-soaking-like phenotype on the lower leaf surface of $N$. benthamiana caused by Lr14a overexpression 2 dpi was suppressed by infiltration of $2 \mathrm{mM}$ Lanthanum(III) chloride $\left(\mathrm{LaCl}_{3}\right)$, a calcium channel blocker ${ }^{34,35}$ two hours after Agrobacterium infiltration. The region infiltrated with $\mathrm{HA}$-tagged $\mathrm{Lr} 14 \mathrm{a}$ coding sequence and $\mathrm{H}_{2} \mathrm{O}$ as control showed a water-soaking-like phenotype, while the region infiltrated with $\mathrm{Lr} 14 a$ and LaCl 3 showed an inhibition of this phenotype. The water-soaking-like phenotype is also detectable under blue light illumination. Source data underlying (c) and (d) are provided as a Source Data file. 
water-soaking ${ }^{33}$ phenotype. This phenotype is presumably caused by a change in the osmotic balance of leaf cells ${ }^{33}$. The same phenotype was also observed with an $\mathrm{N}$-terminal human influenza hemagglutinin (HA) tagged Lr14a coding sequence as well as with an N-terminal eGFP tagged Lr14a coding sequence (Fig. 5c). The eGFP tagged phenotype was weaker, possibly due to the larger tag or differences in expression associated with different vector systems. Infiltration of the Lr14a coding sequence of the EMS loss-of-function mutant (L362F) did not result in the water-soaking-like phenotype (Fig. 5c). Based on the RNAseq data we suggest LR14A directly or indirectly induces changes in calcium ion fluxes at the plasma membrane. Overexpression of Lr14a in N. benthamiana could thus result in an osmotic imbalance, leading to the water-soaking-like phenotype (Fig. 5c). The water-soaking-like phenotype was abolished when Lanthanum(III) chloride $\left(\mathrm{LaCl}_{3}\right)^{34,35}$ was infiltrated two hours after infiltration with $\operatorname{Lr} 14 a$ (Fig. 5 d). $\mathrm{LaCl}_{3}$ is known to be a calcium channel blocker, inhibiting calcium influxes into the cell ${ }^{34,35}$. Taken together, our data suggest the Lr14a-mediated resistance is related to changes of calcium fluxes.

Our results demonstrate the involvement of LR14A, a representative of an ANK-TM protein family, in race-specific disease resistance in wheat. Future studies on LR14A and ACD6 will show if these proteins act as interactors with PRRs, helper NLRs ${ }^{36}$ or possibly as calcium channels at the plasma membrane. The absence of molecular diversity in the Lr14a gene in wild emmer wheat, the domesticated durum landraces and in hexaploid wheat suggests a low selection pressure for diversification of $\operatorname{Lr} 14 a$, possibly because of an indirect recognition of AvrLR14A. Given the race-specific induction of Lr14a by an avirulent P. triticina isolate, it is tempting to speculate that AvrLR14A could be a transcription factor binding to the Lr14a promoter similar to transcription activator-like effector nucleases (TALEN) $)^{37,38}$ in bacteria of the genus Xanthomonas. However, LR14A might also be the direct target of AvrLR14A with the absence of diversity caused by a short evolutionary time since the introgression of the gene into the wheat gene pool. Specifically, the ANK domain could be a direct target of pathogen effectors. Recently, the wheat stripe rust resistance gene $\mathrm{YrU} 1$ was found to encode an NLR protein with an integrated ANK domain ${ }^{39}$. The ANK domain of $Y r U 1$ is derived from ANK-TM proteins and possibly serves as a decoy for pathogen effectors (Supplementary Fig. 6d). Together with our results, this suggests that multiple ANKTM proteins might be involved in race-specific disease resistance in wheat, which forms the basis to exploit this protein family in disease resistance breeding.

\section{Methods}

Plant materials. The bread wheat (Triticum aestivum) cultivars (cv.) Arina and Forno (Swiss winter wheats), and NILs (near isogenic lines) ArinaLrFor (BC2F(512)-85), ArinaLr14a (BC3F9-85-2019) ${ }^{4}$ were used to clone $\mathrm{Lr} 14 a$ (for primers and PCR conditions see Supplementary Data 9). A subset of 158 lines of a RIL (recombinant inbred lines) population (cv. Arina X cv. Forno) $)^{2}$ was genotyped for Lr14a (for primers and PCR conditions see Supplementary Data 9) and phenotyped after infection with leaf rust. A worldwide collection of 99 wheat and nine spelt ( $T$. aestivum subspecies spelta) accessions, available in our lab were genotyped for $\operatorname{Lr} 14 a$ presence and allelic diversity as well as for infection type. In addition, 43 accessions from the Whealbi collection ${ }^{19}, 11$ lines from a previous study 40 of our lab, and 65 wild tetraploid Emmer genotypes (Triticum dicoccoides) from another study ${ }^{17}$ of our lab were genotyped for $\operatorname{Lr} 14 a$ presence and allelic diversity as well as partially for infection type. A Turkish collection kindly provided by Hakan Ozkan, consisting of 201 wild emmer and 71 Triticum durum cultivars and landraces available in his lab were also tested with the $\operatorname{Lr} 14 a$ marker. All these lines are listed in Supplementary Data 1 and 2.
}

EMS mutagenesis. EMS mutagenesis of ArinaLrFor (BC2F5-85) and (BC2F10-85) was performed with a concentration of $0.6 \%$ and $0.45 \%$ EMS (Sigma Aldrich, St. Louis, Missouri, USA), respectively. Seeds were incubated $16 \mathrm{~h}$ in water at $4{ }^{\circ} \mathrm{C}$, dried for $8 \mathrm{~h}$ on filter paper, and incubated for $16 \mathrm{~h}$ with shaking at room temperature in EMS solution. After washing three times for 30, 45, and $60 \mathrm{~min}$, respectively, and for another 30 min under running tap water, seeds were pregerminated on humid filter paper. Three thousand seeds of BC2F5-85 were mutagenized and pre-germinated seeds were propagated in the field. Single spikes of M0 plants were harvested and M1 plants were infected in the field with leaf rust. The selected susceptible candidate mutant lines were analyzed under field infection conditions over four generations. Three mutant lines were repeatedly phenotyped as susceptible and later confirmed to be susceptible at the seedling stage (BC2F585-M5-167, BC2F5-85-M5-983, BC2F5-85-M5-2659). Three thousand seeds of $\mathrm{BC} 2 \mathrm{~F} 10-85$ were mutagenized and seedling stage infections of the M0 progeny (isolated single spike) revealed four independent susceptible mutant lines (BC2F1085-M1-7, BC2F10-85-M1-11, BC2F10-85-M1-67, BC2F10-85-M1-71), from which BC2F10-85-M1-11 could not be propagated to the next generation.

Leaf rust infection. Leaf rust infections at seedling and adult plant stage were performed as described ${ }^{4}$, with Puccinia triticina isolates of a Swiss collection ${ }^{41}$ available in our lab. Briefly, infections at the seedling stage were performed with leaf rust spores stored in tubes in $-80^{\circ} \mathrm{C}$. They were thawed at $42^{\circ} \mathrm{C}$ for $1 \mathrm{~min}$, mixed with FC-43 oil ( $3 \mathrm{M}^{\mathrm{mm}}$ Fluorinert ${ }^{\mathrm{ms}}$ FC-43, $3 \mathrm{M}$ Electronics, Zwijndrecht, Belgium), and then sprayed with a high-pressure air sprayer on humid plants, 10 days after sowing at two-leaf stage. Seeds were sown in soil (Rasenerde $(20 \%$ organic matter, $\mathrm{pH}\left(\mathrm{CaCl}_{2}\right) 6.5,1.4 \mathrm{~g} / \mathrm{L}$ salt content (KCl), filler (DIN EN 12580)), ökohum $\mathrm{GmbH}$, Herrenhof, Switzerland) and watered initially with $2 \mathrm{~L}$ water, mixed with fertilizer (Wuxal ${ }^{\circledast}$ Profi $(2.5 \mathrm{~mL} / \mathrm{L}$ ), Maag Garden, Syngenta, Düsseldorf, Germany) and growth inhibitor $\left(\right.$ Cycocel $^{\circledast}$ Extra $(1 \mathrm{~mL} / \mathrm{L})$, Omya AG, AGRO, Oftringen, Switzerland). After infection, plants dried for $30 \mathrm{~min}$, were then covered with a plastic foil cover to maintain a high level of humidity, and kept in the dark for $24 \mathrm{~h}$. After 9 days of light incubation (60\% humidity, $16 \mathrm{~h} 350 \mu \mathrm{M}$ light, $20^{\circ} \mathrm{C}$, $8 \mathrm{~h}$ dark, $16^{\circ} \mathrm{C}$ ) under the plastic foil cover, leaf rust infected 2 nd leaves where glued on transparent foil and scanned at 720 dots per inch on an Epson Perfection V850 Pro scanner (Epson, Kloten, Switzerland). For scoring, the leaf rust infection phenotypes were evaluated according to the classifications established by McIntosh and colleagues ${ }^{5} .1=$ small uredia with necrosis, $2=$ small to medium sized uredia with necrosis or chlorosis, 3 = medium sized uredia with or without chlorosis, ; = hypersensitive flecks.

Leaf rust infections in the field were performed with a mixture of 16 Swiss $P$. triticina isolates ${ }^{41}$. In spring (March), two weeks old susceptible wheat seedling pots (cv. Walter $60 \%$, cv. Arina $20 \%$, cv. Frisal 20\%) were infected with a leaf rust spore/talcum powder mixture and grown for two weeks in a greenhouse $(60 \%$ humidity, $16 \mathrm{~h} 350 \mu \mathrm{M}$ light, $17^{\circ} \mathrm{C}, 8 \mathrm{~h}$ dark, $\left.13^{\circ} \mathrm{C}\right)$. After two weeks of acclimatization, they were transferred next to the infection rows $(33 \% \mathrm{cv}$. Arina, $33 \% \mathrm{cv}$. Bernina, $33 \% \mathrm{cv}$. Frisal) which are positioned right and left from three rows of testing plants, 40 seeds/row. Three to four replicates of testing plants were randomly placed. The field was located in Reckenholz, Zurich, Switzerland. Scoring was done as soon as leaf rust uredia were visible on flag leaves (summer, mid/end June, depending on climate conditions) and performed for around two weeks every second day until flag leaves dried out. For scoring, coverage of leaf rust uredia on flag leaves was estimated as percentage $e^{42}$ and compared with susceptible and resistant controls.

Chromosome flow sorting. Wheat cv. Arina and ArinaLrFor carry the welldescribed $7 \mathrm{BL} / 5 \mathrm{BL}$ translocation ${ }^{43}$. Translocation chromosome $7 \mathrm{BL} / 5 \mathrm{BL}$ was purified by flow cytometric sorting ${ }^{44}$. Briefly, suspensions of mitotic metaphase chromosomes were prepared from synchronized wheat seedling root tips ${ }^{45}$. A fluorescein isothiocyanate (FITC) conjugate was used to label GAA microsatellites on chromosomes in suspension by FISHIS ${ }^{46}$ while $4^{\prime}, 6$-diamidino-2-phenylindole (DAPI), $2 \mu \mathrm{g} / \mathrm{ml}$, was used to stain chromosomal DNA. The chromosome samples were analyzed at a rate of 2000 chromosomes/s by a FACSAria SORP (BD Biosciences, San Jose, California, USA) and sort windows were set up on FITC versus DAPI dot plots to sort chromosome $7 \mathrm{BL} / 5 \mathrm{BL}$ in wheat. The chromosomes were sorted at rates of $25 / \mathrm{s}$ into $0.5-\mathrm{ml}$ PCR tubes containing $40 \mu \mathrm{l}$ deionized water. For each of the mutants, 25-35 thousand 7BL/5BL chromosomes were sorted, which yielded DNA for three independent multiple displacement amplifications ${ }^{47}$ using the Illustra GenomiPhi V2 DNA amplification kit (GE Healthcare Life Sciences, Pittsburgh, Pennsylvania, USA). To achieve a higher sequence representation, amplified DNA samples derived from each line were pooled. To determine the purity of the 7BL/5BL chromosome in flow-sorted fractions, 2000 chromosomes were sorted into a 5 - $\mu \mathrm{l}$ drop of P5 buffer on a microscope slide during each sort run and sorted chromosomes were identified by FISH using probes for GAA microsatellites and Afa family repeat ${ }^{48}$. Purities of the $7 \mathrm{BL} / 5 \mathrm{BL}$ chromosome and total DNA yields for the three Lr14a mutant lines were as follows: BC2F5-85-M5-167 = $95 \%$ purity and $8.29 \mu \mathrm{g}$ DNA; BC2F5-85-M5-983 = 95\% purity and $8.75 \mu \mathrm{g}$ DNA; BC2F5-85-M5-2659 = 96\% purity and $12.07 \mu \mathrm{g}$ DNA.

Sequencing. Sanger sequencing was performed in house on an ABI 3730 (Thermo Fisher Scientific, Waltham, Massachusetts, USA). Primers use for sequencing are given in Supplementary Data 9. Illumina sequencing library preparation and Illumina sequencing were performed by the Functional Genomics Center Zurich, (https://fgcz.ch/, Zurich, Switzerland). Enriched chromosome 7BL/5BL with a size of $977 \mathrm{Mb}^{9}$ from three ArinaLrFor EMS mutant lines were sequenced on an 
Illumina HiSeq 2500 (Illumina, San Diego, California, USA) to an average coverage of 20 -fold. Whole-genome-de-novo paired-end sequencing of $150 \mathrm{bp}$ DNA fragments was performed.

MutChromSeq with synthetic assembly and synthetic Illumina reads. The flow-sorted chromosome 7B EMS mutant Illumina raw reads were analyzed for their quality using FastQC (http://www.bioinformatics.bbsrc.ac.uk/projects/fastqc). For sequencing adapter removal and quality trimming, cutadapt ${ }^{49}$ and sickle (https://github.com/najoshi/sickle), with the sickle parameter $-\mathrm{q}=25$ and $-1=20$, were used. ArinaLrFor pseudomolecule version 3 7BL (positions 1-422 Mb) as established as part of the recently published $10+$ Wheat Genomes Project ${ }^{9}$ (www.10wheatgenomes.com, https://wheat.ipk-gatersleben.de//) was used for fragmentation into synthetic assemblies with synthetic contig sizes of 3000, 5000, and $7000 \mathrm{bp}$ respectively for three different MutChromSeq runs using the perl command: 'perl -ne 'BEGIN $\{\$ /=$ " $>$ " $\}$ s $/(. *) / / ; \$ n=\$ 1 ; \mathrm{s} / \mathrm{n} / / \mathrm{g} ; \$ \mathrm{i}=0 ; \mathrm{s} /(.\{1, \$\{$ con tig_size\}\})/printf(“>\%s_\%05d $\backslash n \% s \backslash n$ ”, $\$$ n, $++\$ i, \$ 1) ; /$ ge;\}' chromosome7BL.fasta > chromosome7BL \$ contig_size\}.fasta'. Transposable elements were masked in synthetic assemblies using the transposable element database version 2018 (https:// www.botinst.uzh.ch/en/research/genetics/thomasWicker/trep-db.html, http:// botserv2.uzh.ch/kelldata/trep-db/index.html).

As reference, synthetic Illumina reads of $150 \mathrm{bp}$ each, paired-end with an insert size of 2,000 bp, without mutations and a coverage of 20 -fold were synthesized by using the chromosome 7BL pseudomolecule and the dwgsim program (https://github. com/nh13/DWGSIM) with the command: 'dwgsim -e 0 -E 0 -d 2000 -N 2 -C 20 -1 150 -2 150 -r 0 -F 0 -R 0 -X 0 -I 1 -y 0 -n 150 -c 0 -S 2 -q G chromosome7BL.fasta reads_chromosome7BL'.

MutChromSeq was performed three times with synthetic Illumina reads as reference wild-type reads and with synthetic assemblies of 3000, 5000, and $7000 \mathrm{bp}$ synthetic contig length. Commands were used according to protocol (https:// github.com/steuernb/MutChromSeq) ${ }^{10}$. Adjustments were made in Pileup2XML command (pileupfloat $=0.1$, pileupcoverage $=10$ ) and MutChromSeq command (mutchromseqfloat $=0$, mutchromseqcoverage $=8, \mathrm{n}=3, \mathrm{z}=1$ ).

Three different MutChromSeq runs resulted in 25 (3000 bp), 26 (5000 bp), and 30 (7000) synthetic candidate contigs (Supplementary Data 10,11,12) The 25 synthetic candidate contigs of the $3000 \mathrm{bp}$ synthetic assembly were filtered for standard EMS mutations (G/C to A/T) by eye. The remaining three synthetic candidate contigs of the 3000 bp MutChromSeq run (Supplementary Data 13) were blasted for gene content with the Chinese Spring annotation: IWGSC Reference Sequence v1.0 Annotation (https://wheat-urgi.versailles.inra.fr/Seq-Repository/ Annotations) and checked if EMS mutations lead to coding sequence changes in genes. Two of the remaining three synthetic contigs had only short overlaps with protein-coding genes ( $38 \mathrm{bp}$ and $209 \mathrm{bp}$, respectively), while the third one had a hit of $900 \mathrm{bp}$ for a protein-coding gene and also the EMS mutations occurred within the coding sequence.

Virus-induced gene silencing. For virus-induced gene silencing (VIGS) target design, exon 1 and exon 3 of Lr14a were aligned respectively against the recently published ArinaLrFor genome sequence ${ }^{9}$ using BLASTN to find the sequences with the lowest similarity to the rest of the genome. Based on that, two regions with lowest similarities (exon 1, 78-83\% similarity, 213 - 486 bp = VIGS1; exon 3, 84\% similarity, $20-287 \mathrm{bp}=$ VIGS3) were selected as VIGS target sequences.

VIGS cloning and infection were performed as described ${ }^{50-53}$, using a phytoene desaturase gene silencing construct to induce photo bleaching, as positive control. For cloning the VIGS1 or VIGS3 amplicon (primers used are listed in Supplementary Data 9) into the vector pBS-BSMV- $\gamma$, respectively, the restriction sites NotI and PacI in antisense direction were used. The virus genome is composed of the pBS-BSMV- $\alpha$, pBS-BSMV- $\beta$, and pBS-BSMV- $\gamma$ plasmids, where the $\gamma$ plasmid carried the VIGS target sequence VIGS1 or VIGS3. The wild-type (WT) viral genome was used as control. For in vitro synthesis of virus RNA, the Invitrogen $^{\text {nx }}$ mMESSAGE mMACHINE ${ }^{\text {sx }}$ T7 Transcription Kit (Thermo Fisher Scientific, Waltham, Massachusetts, USA) was used. For virus infection, seeds in soil were kept at $4{ }^{\circ} \mathrm{C}$ for 7 days. Then, seedlings were placed in a growth chamber $\left(60 \%\right.$ humidity, $16 \mathrm{~h} 350 \mu \mathrm{M}$ light, $23^{\circ} \mathrm{C}, 8 \mathrm{~h} \mathrm{dark}, 16^{\circ} \mathrm{C}$ ). Seedlings at this time were at the two-leaf stage and the first leaf was completely inoculated with viral RNA. At day 14 after virus infection, a seedling leaf rust infection was performed and 10 days later, leaf rust phenotypes on the 3rd and/or 4th leaf were documented. Leaf rust uredia and decreased hypersensitive flecks count was done in a $0.5 \times 2 \mathrm{~cm}$ square in the middle of leaves from one representative experimental repetition ( $n$ $=8,7,6$ leaves for VIGS1, VIGS3, WT, respectively).

Bioinformatics analysis. The programs Dotter, Water, Clustalx, Clustalw, MrBayes, FigTree, and blast were all obtained from Ubuntu repositories (ubuntu. com). Analysis of sequenced PCR products was performed with CLC Main Workbench 20.0.2 and versions below. Ankyrin (ANK) repeats were annotated manually through dot plots of the predicted LR14A protein against itself and by identifying previously described conserved ANK amino acid motifs ${ }^{12,54}$. The threedimensional structure of the LR14A ANK domain was predicted with RaptorX ${ }^{55}$, (http://raptorx.uchicago.edu/), Phyre2 ${ }^{56}$, (http://www.sbg.bio.ic.ac.uk/ phyre2/ $\mathrm{html} /$ page.cgi?id=index), and HHPred ${ }^{57}$, (https://toolkit.tuebingen.mpg.de/tools/ hhpred). Transmembrane domains were predicted with TMHMM server $\mathrm{v} 2.0$ (http://www.cbs.dtu.dk/services/TMHMM/).

Molecular dating. Regions homologous to the Lr14a locus in bread wheat cv. Lancer and Spelt wheat PI190962 were identified by blastn using the coding sequence of $\operatorname{Lr} 14 a$ as query. In the case of bread wheat cv. Chinese Spring (which does not contain $\operatorname{Lr} 14 a$ ), the Lr14a region was identified by aligning the homologous regions of chromosome $7 \mathrm{~B}$ in the two cultivars. The regions containing the gene and $200 \mathrm{~kb}$ of flanking sequence were extracted from the pseudomolecule sequences and aligned with Dotter in order to determine the extent of the alignable sequences. Once boundaries of conserved sequences were established, homologous regions were aligned with the emboss program Water (https://www.ebi.ac.uk/ Tools/psa/emboss_water/). For these alignments, only intergenic sequences (sequences $>500 \mathrm{bp}$ up- or downstream from annotated genes) were used. Divergence time calculations between haplotypes were performed using a substitution rate of $1.3 \mathrm{E}-8$ substitutions per site per year ${ }^{58,59}$. We only used nucleotide substitutions for molecular dating, and insertions and deletions were ignored ${ }^{58}$.

Collinearity breakpoint between the Lr14a locus in ArinaLrFor and Chinese Spring. The Lr14a locus is fragmented to different degrees in the genome assemblies of bread wheat cultivars ArinaLrFor, Lancer, Chinese Spring, and spelt wheat accession PI190962. Because comparison of Chinese Spring and ArinaLrFor was central to our model for the evolution of the Lr14a locus, the breakpoints of sequence collinearity between Chinese Spring and ArinaLrFor were confirmed by PCR. Here, the precise end points of sequence collinearity were determined with the program Dotter. Subsequently, primer pairs (primers are listed in Supplementary Data 9) were generated to bridge the breakpoints in ArinaLrFor. Sequenced PCR fragments aligned to the genomic sequences could confirm the integrity of the sequences in the breakpoint regions.

Phylogenetic analysis. Proteins with the ANK-TM domain organization form a very large family with dozens of members in all angiosperm genomes. To reduce complexity and shorten computation time, three types of LR14A homologs were selected as follows: 1 . the top ten hits of a blastp search of LR14A against Arabidopsis proteins, 2. the top ten hits of a blastp search of LR14A against all proteins of the wheat (cv. Chinese Spring) B genome, and 3. the top ten hits of a blastp search of Arabidopsis ACD6 against all proteins of the wheat (cv. Chinese Spring) $\mathrm{B}$ genome. The last search was performed to identify the closest homologs of ACD6 in wheat. The wheat dataset was reduced to the B genome to simplify the tree and shorten computation time. ANK-TM-type proteins that were previously described to be involved in plant stress response were added to the selection. These included ACD6 ${ }^{19,24,60}, \mathrm{BDA}^{21}$, and ITN $1^{20}$ from Arabidopsis and ZmACD6 ${ }^{22}$ from maize. Protein sequences were aligned with Clustalw (obtained from ubuntu.com repositories) using a gap opening penalty of 5 and a gap extension penalty of 0.01 . The multiple alignments were converted to nexus format with Clustalx (obtained from ubuntu.com repositories). A phylogenetic tree for LR14A homologs was constructed with MrBayes ${ }^{61}$, using parameters lset $=6$ and rates $=$ invgamma and by running Markov chain Monte Carlo simulation $(\mathrm{mcmc})$ for 700,000 generations with a sample frequency of 10 . Trees were summarized using a burn-in of $25 \%$. The consensus tree was visualized with FigTree (http://tree.bio.ed.ac.uk/software/ figtree/). The phylogenetic analysis of the ANK domains of the proteins was done in the same way as for the full-length proteins described above. The difference was that the ANK domain of $\mathrm{YrUl}^{39}$ was added to the datasets and that the $\mathrm{mcmc}$ simulation of MrBayes was run for 1,000,000 generations. The length of the ANK domain used for the analysis was determined by aligning YrU1 with LR14A homologs.

LR14A localization. The coding sequence of $L r 14 a$ was cloned (primers are listed in Supplementary Data 9) with the Invitrogen ${ }^{\mathrm{nw}} \mathrm{pENTR}^{\mathrm{m}} \mathrm{D}$-Topo ${ }^{\oplus}$ Cloning Kit and Invitrogen $^{\mathrm{mw}}$ Gateway $^{\oplus}$ Lr Clonase ${ }^{\mathrm{mt}}$ II Plus Enzyme Mix (Thermo Fisher Scientific, Waltham, Massachusetts, USA) into the pGWB506 vector (pGWB506 was a gift from Tsuyoshi Nakagawa (Addgene plasmid \# 74848; http://n2t.net/ addgene:74848; RRID:Addgene_74848) ${ }^{62}$ ) to attach an N-terminal enhanced green fluorescence protein (eGFP) to LR14A, expressed by a cauliflower mosaic virus (CaMV) $35 \mathrm{~S}$ promotor. As plasma membrane marker, an $\mathrm{N}$-terminal monomeric red fluorescence protein (mRFP) tagged AtREM1.2 (At3g61260) coding sequence under a CaMV $35 \mathrm{~S}$ promotor was used ${ }^{63}$. Live cell imaging was performed using a Leica SP5 confocal laser scanning microscopy system (Leica, Wetzlar, Germany) equipped with Argon, DPSS, and He-Ne lasers and hybrid detectors and the LAS AF Version 2.7.3.9723 Leica Microsystems CMS GmbH Software. Imaging was performed as previously described ${ }^{64}$, with minor modifications. Briefly, Nicotiana benthamiana transformation, with Agrobacterium tumefaciens (strain GV3101), carrying the plasmids of interest as transformation vector, was performed as described ${ }^{65}$. Three days after N. benthamiana infiltration, leaf samples of $5 \times 5 \mathrm{~mm}$ were transferred between a glass slide and a cover slip in a drop of water. For plasmolysis induction, samples were incubated $10-15 \mathrm{~min}$ in $4 \% \mathrm{NaCl}$ prior to imaging. Fluorescence was observed with excitation wavelengths of $488 \mathrm{~nm}$ and emission wavelengths of 490 to $550 \mathrm{~nm}$ for eGFP and with excitation wavelengths of $561 \mathrm{~nm}$ and emission wavelengths of 580-640 nm for mRFP. Chlorophyll 
autofluorescence was captured using emission wavelengths of 700-720 nm after excitation of the samples at $488 \mathrm{~nm}$. Fluorescence intensities across plasma membrane sections were measured using the plot line plugin in Fiji software (https://fiji.sc/). Experiments were performed using strictly identical confocal acquisition parameters (e.g. laser power, gain, zoom factor, resolution, and emission wavelengths reception), with detector settings optimized for low background and no pixel saturation. Pseudo-colored images were obtained using "Green", "Magenta", and "Turquoise" look-up-table (LUT) of Fiji software.

RNAseq experiments and analysis. RNA was extracted with the SV Total RNA Isolation System (Promega, Madison, Wisconsin, USA). N. benthamiana leaves were transiently transformed with A. tumefaciens (strain GV3101), carrying the plasmids of interest as described ${ }^{65}$. Leaves were transiently transformed with either a human influenza hemagglutinin (HA) N-terminal tagged Lr14a coding sequence carrying vector pIPKb00466, or an empty vector, both co-infiltrated with $A$. tumefaciens carrying a P19 vector (P19 protein from tomato bushy virus (TBSV) ${ }^{67}$ ) sampled $27 \mathrm{hpi}$ (hours post-inoculation). Wheat leaves inoculated with $P$. triticina isolates 96209 ( $L r 14 a$ avirulent isolate), 95037 (Lr14a virulent isolate) or infection medium, respectively, were sampled 8 days post-inoculation. $N$. benthamiana RNA samples were sequenced on a Novaseq PE150 (Illumina, San Diego, California, USA) to a total data amount of $6 \mathrm{Gbp} / 20 \mathrm{M}$ reads per sample. Wheat samples were sequenced on a Novaseq 6000 S4 (Illumina, San Diego, California, USA) to a total data amount of $12 \mathrm{Gbp} / 40 \mathrm{M}$ reads per sample.

In order to quantify the transcriptomic response induced by the transient expression of Lr14a in N. benthamiana, we used the RNA-Seq analysis pipeline Salmon ${ }^{68}$ with standard parameters. RNAseq libraries of $N$. benthamiana plants in the presence or absence of $\operatorname{Lr} 14 a$ at $27 \mathrm{~h}$ post-infiltration were quantified in mapping-based mode on the $N$. benthamiana coding sequence annotation (version 1.0.1). The estimated number of mapped reads were further considered for differential expression (DE) analysis. We used the R package edge $\mathrm{R}^{69}$ and only the genes with a $\log 2 \mathrm{FC}>|1.5|$ and an adjusted $p$-value $(\mathrm{FDR})<0.05$ were considered as differentially expressed ${ }^{70}$. To further investigate the interactions between $N$. benthamiana genes that were up-regulated by the transient expression of $\operatorname{Lr} 14 a$, we performed a gene enrichment analyses against the Gene Ontology (GO) database. GO terms enriched in up-regulated genes were identified using the R package GOseq ${ }^{71}$, and the results with a corrected $p$-value smaller than 0.05 were considered significant. AgriGO v2.072 was finally used to generate the directed acyclic graph (DAG) of the GO molecular functions. The same workflow was used for analyzing the wheat transcriptomic data. Coding sequences and corresponding GO identifiers, blast homologs and gene functions, were retrieved from the International Wheat Genome Sequencing Consortium Reference Sequence (IWGSC RefSeq) v1.0 annotation.

Finally, the wheat transcriptomic response induced by LR14A was compared with other RNA-Seq studies in which wheat plants were challenged with different abiotic and biotic stresses. For this purpose, we only considered the DEGs that were identified between ThatcherLr14a and Thatcher, upon infection of the avirulent $P$. triticina isolate at $8 \mathrm{dpi}$. The transcriptomic data were downloaded from wheatexpression.com ${ }^{70,73,74}$ and the following stress treatments were considered: Zymoseptoria tritici (Zt; ERP009837), Fusarium graminearum (Fg; ERP013829), stripe rust and wheat powdery mildew (Sr, Pm; ERP013983, SRP041017), Fusarium pseudograminearum (Fp; SRP048912), PAMPs (chitin and flg22) and cold (SRP043554). Differential gene expression analysis was performed as mentioned above and the R package pheatmap was used for comparing the log2FC values of the LR14A-induced DEGs across all the different stress treatments. In order to improve the data visualization, $\log 2 \mathrm{FC}$ values were scaled to $\mathrm{Z}$-scores with the argument scale $=$ 'row'.

Lr14a phenotyping and $\mathrm{LaCl}_{3}$ phenotype inhibition on Nicotiana benthamiana lower leaf surface. A. tumefaciens carrying an overexpression construct for wildtype (WT) Lr14a coding sequence in the vector PIPKb00466, EMS loss of function mutant (L362F) Lr14a coding sequence in the vector pIPKb004 ${ }^{66}$, N-terminal enhanced green fluorescence protein (eGFP) tagged Lr14a coding sequence in a pGWB506 vector (pGWB506 was a gift from Tsuyoshi Nakagawa (Addgene plasmid \# 74848; http://n2t.net/addgene:74848; RRID:Addgene_74848) ${ }^{62}$ ) and Nterminal human influenza hemagglutinin (HA) tagged Lr14a coding sequence in the vector pIPKb004 ${ }^{66}$ was used to transiently transform $N$. benthamiana as described ${ }^{65}$. Lanthanum (III) chloride $\left(\mathrm{LaCl}_{3}\right)$ was infiltrated $1-2 \mathrm{~h}$ post Agrobacteria infiltration in a concentration of $2 \mathrm{mM}$, dissolved in water. Water was used for infiltration on control leaf spots. Leaves were scanned 2 or 3 days postinoculation on an Epson Perfection V850 Pro scanner (Epson, Kloten, Switzerland), or under a Fusion FX Imaging System (Vilber Lourmat, Eberhardzell, Germany) as described for hypersensitive response (HR) measurements ${ }^{65}$.

Salicylic acid extractions and measurements. Salicylic acid extractions and measurements were performed as previously described ${ }^{75}$. Briefly, ArinaLrFor WT and mutant plants were infected 10 days after germination with the avirulent $P$. triticina isolate 96209 and leaf samples were taken 8 dpi. SA quantification was performed using ultra-high performance liquid chromatography-tandem mass spectrometry (UHPLC-MS/MS). Fresh frozen tissues were ground in liquid nitrogen and approximately $100 \mathrm{mg}$ of powder was weighed in a microcentrifuge tube. To this tube $990 \mu \mathrm{L}$ of ethylacetate:formic acid (99.5:0.5, v/v), $1 \mathrm{ng}$ of SA-d4 and 3-5 glass beads were added, and the tube was shaken at $30 \mathrm{~Hz}$ for $3 \mathrm{~min}$ in a mixer mill. After centrifugation, the supernatant was reserved and the pellet re-extracted with $0.5 \mathrm{~mL}$ of extraction solvent. The supernatants were combined, evaporated and the dry residue was reconstituted in $100 \mu \mathrm{L}$ of methanol $70 \%$. A $5 \mu \mathrm{L}$ injection was made in the UHPLC-MS/MS system which was composed of a Dionex Ultimate 3000 RSLC (Thermo Fisher Scientific, Waltham, Massachusetts, USA) coupled to a 4000 QTRAP (AB Sciex, Framingham, Massachusetts, USA). A gradient separation was performed on a $50 \times 2.1 \mathrm{~mm}$ Acquity UPLC BEH C18 column (Waters, Milford, Massachusetts, USA) using mobile phases of water and acetonitrile, both supplemented with $0.05 \%$ formic acid. The mass spectrometer was set in negative electrospray ionization and the transitions $137 / 93$ and 141/97 were monitored for SA and SA-d4, respectively.

Molecular methods to map, clone, and characterize Lr14a. Genomic wheat DNA was extracted following the Cetyltrimethylammonium bromide (CTAB) extraction protocol ${ }^{76}$, using cold dichlormethane:isoamylalcohol (24:1) instead of phenol:chloroform:isoamylalcohol (48:48:4). Genotyping for Lr14a was performed using primers and PCR conditions listed in Supplementary Data 9. PCR amplification of the Lr14a gene (for primers and conditions see Supplementary Data 9) had to be performed in two fragments, as microsatellite repeats in the first intron blocked polymerase activity and a full-length amplification of the Lr14a gene was not possible. For mRNA extraction of infected 2nd leaves, the Invitrogen $^{\mathrm{mw}}$ Dynabeads ${ }^{\mathrm{m}}$ mRNA DIRECT ${ }^{\mathrm{mw}}$ Kit (Thermo Fisher Scientific, Waltham, Massachusetts, USA) was used. The mRNA integrity was validated by gel electrophoresis as well as spectrophotometric analysis using a NanoDrop 1000 Spectrophotometer (Thermo Fisher Scientific, Waltham, Massachusetts, USA). The DNA Ladder used was GeneRULER ${ }^{\mathrm{m}} 1 \mathrm{~kb}$ Plus DNA Ladder (Thermo Fisher Scientific, Waltham, Massachusetts, USA). The mRNA was used to synthesize cDNA with the iScript Advanced cDNA Synthesis Kit for RT-qPCR (BIO-RAD, Hercules, California, USA). Sequencing of cDNA of Lr14a (primers are listed in Supplementary Data 9), extracted from leaf rust infected leaves revealed a single cDNA version. Therefore, we concluded that no alternative splicing variants are produced. For race PCR (primers are listed in Supplementary Data 9) the SMARTer ${ }^{\circledR}$ RACE $5^{\prime} / 3^{\prime}$ Kit (Takara Bio Europe, SaintGermain-en-Laye, France) was used. Amplification of the $5^{\prime}$ and $3^{\prime}$ untranslated region (UTR) revealed no variations in UTR length. Generated cDNA was also used for qPCR (primers are listed in Supplementary Data 9) using the KAPA SYBR $^{\circledast}$ FAST qPCR Master Mix (2X) Kit (Sigma Aldrich, St. Louis, Missouri, USA). Three technical replicates each of three biological replicates were used for qPCR. In addition, pathogenesis response marker genes $(P R 1,2,5)^{15}$ (primers are listed in Supplementary Data 9) were used for qPCR characterization of ArinaLrFor WT and mutant lines.

Reporting summary. Further information on research design is available in the Nature Research Reporting Summary linked to this article.

\section{Data availability}

All data supporting the findings of this work are available within the paper and its supplementary information files. A reporting summary for this article is available as a supplementary information file. The datasets and plant materials generated and analyzed during the current study are available from the corresponding author upon request. Sequence data were deposited at the NCBI GenBank under the accession number MT123593 (Lr14a coding sequence), and at the NCBI sequence read archive (SRA) database under the accession number PRJNA529355 (flow-sorted chromosome 7B of three ArinaLrFor Lr14a EMS mutants) or are available via the recently published 10+ Wheat Genome Project ${ }^{9}$ [www.10wheatgenomes.com or https://wheat.ipk-gatersleben. de//]. RNAseq raw data were deposited at the NCBI SRA database under the accession number PRJNA674985 for Triticum aestivum or PRJNA674843 for Nicotiana benthamiana. Source data are provided with this paper.

Received: 4 June 2020; Accepted: 18 December 2020; Published online: 11 February 2021

\section{References}

1. Kolmer, J. Leaf Rust of Wheat: Pathogen Biology, Variation and Host Resistance. Forests 4, 70-84 (2013).

2. Schnurbusch, T. et al. Dissection of quantitative and durable leaf rust resistance in Swiss winter wheat reveals a major resistance QTL in the Lr34 chromosomal region. Theor. Appl. Genet. 108, 477-484 (2004).

3. Lagudah, E. S. et al. Gene-specific markers for the wheat gene Lr34/Yr18/ Pm38 which confers resistance to multiple fungal pathogens. Theor. Appl. Genet. 119, 889-898 (2009).

4. Singla, J. et al. Characterization of Lr75: a partial, broad-spectrum leaf rust resistance gene in wheat. Theor. Appl .Genet. 130, 1-12 (2017). 
5. McIntosh, R. A., Wellings, C. R. \& Park, R. F. Wheat Rusts: an Atlas of Resistance Genes (Springer, Netherlands, 1995).

6. Dyck, P. \& Johnson, R. Temperature sensitivity of genes for resistance in wheat to Puccinia recondita. Can. J. Plant Pathol. 5, 229-234 (1983).

7. Dyck, P. L. \& Samborski, D. J. The genetics of two alleles for leaf rust resistance at the Lr14 locus in wheat. Can. J. Genet. Cytol. 12, 689-694 (1970).

8. Law, C. N. \& Johnson, R. A genetic study of leaf rust resistance in wheat. Can. J. Genet. Cytol. 9, 805-822 (1967).

9. Walkowiak, S. et al. Multiple wheat genomes reveal global variation in modern breeding. Nature 588, 277-283 (2020).

10. Sanchez-Martin, J. et al. Rapid gene isolation in barley and wheat by mutant chromosome sequencing. Genome Biol. 17, 221 (2016).

11. Mosavi, L. K., Cammett, T. J., Desrosiers, D. C. \& Peng, Z. Y. The ankyrin repeat as molecular architecture for protein recognition. Protein Sci. 13, $1435-1448$ (2004).

12. Li, J., Mahajan, A. \& Tsai, M. D. Ankyrin repeat: a unique motif mediating protein-protein interactions. Biochemistry 45, 15168-15178 (2006).

13. Vo, K. T. X. et al. Molecular insights into the function of ankyrin proteins in plants. J. Plant Biol. 58, 271-284 (2015).

14. Singh, R. P. et al. Disease impact on wheat yield potential and prospects of genetic control. Annu. Rev. Phytopathol. 54, 303-322 (2016).

15. Zhang, W. et al. Identification and characterization of $\mathrm{Sr} 13$, a tetraploid wheat gene that confers resistance to the Ug99 stem rust race group. Proc. Natl Acad. Sci. 114, E9483-E9492 (2017).

16. Marone, D. et al. Genetic analysis of durable resistance against leaf rust in durum wheat. Mol. Breed. 24, 25-39 (2009).

17. Yahiaoui, N., Kaur, N. \& Keller, B. Independent evolution of functional Pm3 resistance genes in wild tetraploid wheat and domesticated bread wheat. Plant J. 57, 846-856 (2009).

18. McFadden, E. S. A successful transfer of emmer characters to Vulgare wheat. Agron. J. 12, 1020-1034 (1930)

19. Dong, X. The role of membrane-bound ankyrin-repeat protein ACD6 in programmed cell death and plant defense. Sci. STKE 2004, pe6 (2004).

20. Sakamoto, H., Matsuda, O. \& Iba, K. ITN1, a novel gene encoding an ankyrinrepeat protein that affects the ABA-mediated production of reactive oxygen species and is involved in salt-stress tolerance in Arabidopsis thaliana. Plant $\mathrm{J}$. 56, 411-422 (2008).

21. Yang, Y. et al. The ankyrin-repeat transmembrane protein BDA1 functions downstream of the receptor-like protein SNC2 to regulate plant immunity. Plant Physiol. 159, 1857-1865 (2012).

22. Zhang, Z., Guo, J., Zhao, Y. \& Chen, J. Identification and characterization of maize ACD6-like gene reveal ZmACD6 as the maize orthologue conferring resistance to Ustilago maydis. Plant Signal. Behav. 14, e1651604 (2019).

23. Todesco, M. et al. Natural allelic variation underlying a major fitness trade-off in Arabidopsis thaliana. Nature 465, 632-636 (2010).

24. Lu, H., Liu, Y. \& Greenberg, J. T. Structure-function analysis of the plasma membrane-localized Arabidopsis defense component ACD6. Plant J. 44, 798-809 (2005).

25. Zhang, Z., Shrestha, J., Tateda, C. \& Greenberg, J. T. Salicylic acid signaling controls the maturation and localization of the arabidopsis defense protein ACCELERATED CELL DEATH6. Mol. Plant 7, 1365-1383 (2014).

26. Tateda, C. et al. Salicylic acid regulates Arabidopsis microbial pattern receptor kinase levels and signaling. Plant Cell 26, 4171-4187 (2014).

27. Lu, H., Rate, D. N., Song, J. T. \& Greenberg, J. T. ACD6, a novel ankyrin protein, is a regulator and an effector of salicylic acid signaling in the Arabidopsis defense response. Plant Cell 15, 2408-2420 (2003).

28. Suo, Y. et al. Structural insights into electrophile irritant sensing by the human TRPA1 channel. Neuron 105, 882-894 e5 (2020).

29. Leng, L. et al. A subclass of HSP70s regulate development and abiotic stress responses in Arabidopsis thaliana. J. Plant Res 130, 349-363 (2017).

30. La Verde, V., Dominici, P. \& Astegno, A. Towards understanding plant calcium signaling through calmodulin-like proteins: a biochemical and structural perspective. Int. J. Mol. Sci. 191331 (2018).

31. Tuteja, N. \& Mahajan, S. Calcium signaling network in plants: an overview. Plant Signal Behav. 2, 79-85 (2007).

32. Dodd, A. N., Kudla, J. \& Sanders, D. The language of calcium signaling. Annu. Rev. Plant Biol. 61, 593-620 (2010).

33. Pitino, M., Allen, V. \& Duan, Y. LasDelta5315 effector induces extreme starch accumulation and chlorosis as Ca. Liberibacter asiaticus infection in Nicotiana benthamiana. Front Plant Sci. 9, 113 (2018).

34. Zhou, B. \& Guo, Z. Calcium is involved in the abscisic acid-induced ascorbate peroxidase, superoxide dismutase and chilling resistance in Stylosanthes guianensis. Biol. Plant. 53, 63-68 (2009).

35. Lehtonen, J. The significance of $\mathrm{Ca} 2+$ in the morphogenesis of Micrasterias studied with EGTA, verapamil, $\mathrm{LaCl} 3$ and calcium ionophore A 23187. Plant Sci. Lett. 33, 53-60 (1984)
36. Zhu, W. et al. Modulation of ACD6 dependent hyperimmunity by natura alleles of an Arabidopsis thaliana NLR resistance gene. PLoS Genet. 14, e1007628 (2018).

37. Bogdanove, A. J. \& Voytas, D. F. TAL effectors: customizable proteins for DNA targeting. Science 333, 1843-1846 (2011).

38. Boch, J. et al. Breaking the code of DNA binding specificity of TAL-type III effectors. Science 326, 1509-1512 (2009).

39. Wang, H., Zou, S., Li, Y., Lin, F. \& Tang, D. An ankyrin-repeat and WRKYdomain-containing immune receptor confers stripe rust resistance in wheat. Nat. Commun. 11, 1353 (2020).

40. Loutre, C. et al. Two different CC-NBS-LRR genes are required for Lr10mediated leaf rust resistance in tetraploid and hexaploid wheat. Plant J. 60, 1043-1054 (2009).

41. Messmer, M. M. et al. Genetic analysis of durable leaf rust resistance in winter wheat. Theor. Appl. Genet. 100, 419-431 (2000).

42. Peterson, R. F., Campbell, A. B. \& Hannah, A. E. A Diagrammatic scale for estimating rust intensity on leaves and stems of cereals. Can. J. Res. C: Botanical Sci. 26, 496-49 (1948).

43. Dolezel, J. et al. Chromosomes in the flow to simplify genome analysis. Funct. Integr. Genomics 12, 397-416 (2012).

44. Kubalakova, M., Vrana, J., Cihalikova, J., Simkova, H. \& Dolezel, J. Flow karyotyping and chromosome sorting in bread wheat (Triticum aestivum L.). Theor. Appl Genet 104, 1362-1372 (2002).

45. Vrana, J. et al. Flow sorting of mitotic chromosomes in common wheat (Triticum aestivum L.). Genetics 156, 2033-2041 (2000).

46. Giorgi, D. et al. FISHIS: fluorescence in situ hybridization in suspension and chromosome flow sorting made easy. PLoS ONE 8, e57994 (2013).

47. Simkova, H. et al. Coupling amplified DNA from flow-sorted chromosomes to high-density SNP mapping in barley. BMC Genomics 9, 294 (2008).

48. Kubalakova, M. et al. Analysis and sorting of rye (Secale cereale L.) chromosomes using flow cytometry. Genome 46, 893-905 (2003).

49. Martin, M. Cutadapt removes adapter sequences from high-throughput sequencing reads. EMBnet. J. 17, 10 (2011).

50. Bhullar, N. K. \& Keller, B. in (Pankaj K. Bhowmik Saikat K. Basu Aakash Goyal (eds.)) Advances in Biotechnology 185-203 (Bentham Science, 2009).

51. Holzberg, S., Brosio, P., Gross, C. \& Pogue, G. P. Barley stripe mosaic virusinduced gene silencing in a monocot plant. Plant J. 30, 315-327 (2002).

52. Scofield, S. R. \& Brandt, A. S. Virus-induced gene silencing in hexaploid wheat using barley stripe mosaic virus vectors. Methods Mol. Biol. 894, 93-112 (2012).

53. Scofield, S. R., Huang, L., Brandt, A. S. \& Gill, B. S. Development of a virusinduced gene-silencing system for hexaploid wheat and its use in functional analysis of the Lr21-mediated leaf rust resistance pathway. Plant Physiol. 138, 2165-2173 (2005).

54. Mosavi, L. K., Minor, D. L. Jr \& Peng, Z. Y. Consensus-derived structural determinants of the ankyrin repeat motif. Proc. Natl Acad. Sci. USA 99, 16029-16034 (2002).

55. Kallberg, M. et al. Template-based protein structure modeling using the RaptorX web server. Nat. Protoc. 7, 1511-1522 (2012).

56. Kelley, L. A., Mezulis, S., Yates, C. M., Wass, M. N. \& Sternberg, M. J. The Phyre2 web portal for protein modeling, prediction and analysis. Nat. Protoc. 10, 845-858 (2015)

57. Zimmermann, L. et al. A completely reimplemented MPI bioinformatics toolkit with a New HHpred server at its core. J. Mol. Biol. 430, 2237-2243 (2018).

58. Buchmann, J. P., Matsumoto, T., Stein, N., Keller, B. \& Wicker, T. Interspecies sequence comparison of Brachypodium reveals how transposon activity corrodes genome colinearity. Plant J. 71, 550-563 (2012).

59. Ma, J. \& Bennetzen, J. L. Rapid recent growth and divergence of rice nuclear genomes. Proc. Natl Acad. Sci. USA 101, 12404-12410 (2004).

60. Rate, D. N., Cuenca, J. V., Bowman, G. R., Guttman, D. S. \& Greenberg, J. T. The gain-of-function Arabidopsis acd6 mutant reveals novel regulation and function of the salicylic acid signaling pathway in controlling cell death, defenses, and cell growth. Plant Cell 11, 1695-1708 (1999).

61. Ronquist, F. et al. MrBayes 3.2: efficient Bayesian phylogenetic inference and model choice across a large model space. Syst. Biol. 61, 539-542 (2012).

62. Nakagawa, T. et al. Improved Gateway binary vectors: high-performance vectors for creation of fusion constructs in transgenic analysis of plants. Biosci. Biotechnol. Biochem. 71, 2095-2100 (2007).

63. Bucherl, C. A. et al. Plant immune and growth receptors share common signalling components but localise to distinct plasma membrane nanodomains. Elife 6, e25114 (2017).

64. Gronnier, J. et al. Structural basis for plant plasma membrane protein dynamics and organization into functional nanodomains. Elife 6, e26404 (2017).

65. Bourras, S. et al. The AvrPm3-Pm3 effector-NLR interactions control both race-specific resistance and host-specificity of cereal mildews on wheat. Nat. Commun. 10, 2292 (2019). 
66. Himmelbach, A. et al. A set of modular binary vectors for transformation of cereals. Plant Physiol. 145, 1192-1200 (2007).

67. Voinnet, O., Pinto, Y. M. \& Baulcombe, D. C. Suppression of gene silencing: a general strategy used by diverse DNA and RNA viruses of plants. Proc. Natl Acad. Sci. USA 96, 14147-14152 (1999).

68. Patro, R., Duggal, G., Love, M. I., Irizarry, R. A. \& Kingsford, C. Salmon provides fast and bias-aware quantification of transcript expression. Nat. Methods 14, 417-419 (2017).

69. Robinson, M. D., McCarthy, D. J. \& Smyth, G. K. edgeR: a Bioconductor package for differential expression analysis of digital gene expression data. Bioinformatics 26, 139-140 (2010).

70. Praz, C. R. et al. Non-parent of origin expression of numerous effector genes indicates a role of gene regulation in host adaption of the hybrid triticale powdery mildew pathogen. Front Plant Sci. 9, 49 (2018).

71. Young, M. D., Wakefield, M. J., Smyth, G. K. \& Oshlack, A. goseq: gene ontology testing for RNA-seq datasets. R. Bioconductor 8, 1-25 (2012).

72. Tian, T. et al. agriGO v2.0: a GO analysis toolkit for the agricultural community, 2017 update. Nucleic Acids Res. 45, W122-W129 (2017).

73. Ramirez-Gonzalez, R. H. et al. The transcriptional landscape of polyploid wheat. Science 361, eaar6089 (2018).

74. Ma, X., Keller, B., McDonald, B. A., Palma-Guerrero, J. \& Wicker, T. Comparative transcriptomics reveals how wheat responds to infection by Zymoseptoria tritici. Mol. Plant Microbe Interact. 31, 420-431 (2018).

75. Glauser, G., Vallat, A. \& Balmer, D. in (David B. Collinge (ed.)) Arabidopsis Protocols (Springer Protocols, 2014).

76. Clarke, J. D. Cetyltrimethyl ammonium bromide (CTAB) DNA miniprep for plant DNA isolation. Cold Spring Harb. Protoc. 2009, pdb.prot5177 (2009).

77. Lang-Pauluzzi, I. The behaviour of the plasma membrane during plasmolysis: a study by UV microscopy. J. Microsc. 198, 188-198 (2000).

\section{Acknowledgements}

The authors would like to thank Gerhard Herren, Lukas Kunz, Luisa K. Schaefer, Esther Jung, Coraline R. Praz, Christoph Stritt, Beatrice Manser, Marion C. Müller, Jonatan Isaksson, Christoph Ringli, Patrick Ackermann, Victoria Widrig, Hanieh Jozevaziri, Jonas Stehlin, Matthias Heuberger, Stefan Lindner, Stephanie Bräunlich, Celia Baroux, and Clara Sanchez for helpful discussions, technical support, and assistance in acquiring necessary techniques and knowledge used in this study. We thank Jan Vrána, Zdeňka Dubská, Romana Šperková, and Jitka Weiserová for the assistance with chromosome sorting and preparation of chromosomal DNA. We would like to thank Hakan Ozkan for information on the Hevidi and Menceki genotypes and provision of diverse durum and wild emmer genotypes, Robert A. McIntosh for discussions about Lr14a resistance phenotypes, Detlef Weigel for discussions about ACD6, the John Innes Centre Germplasm Resource Unit (GRU), Norwich, UK for providing Creso seeds and the gene bank of the Crop Research Institute, Prague, Czech Republic for providing Yaroslav emmer seeds. This project has received funding from the European Union's Horizon 2020 research and innovation program under the Marie Skłodowska-Curie grant agreement No. 674964 , from grant 310030B_182833 by the Swiss National Science Foundation to B Keller, as well as funding by the University of Zurich, and the King Abdullah University of Science and Technology. H.S.., M.K., and J.D. were supported from ERDF project
"Plants as a tool for sustainable global development" (No. CZ.02.1.01/0.0/0.0/16_019/ 0000827). J.G. and P.K. were funded by the European Research Council under the Grant Agreement no. 773153 (grant "IMMUNO-PEPTALK" to C.Z.). J.G. was also supported by a Long-Term Post-Doctoral Fellowship from the European Molecular Biology Organization (EMBO 438-2018).

\section{Author contributions}

M.C.K., T.W., S.G.K., and B.K. designed the research; M.C.K. performed experiments and analyzed the data; J.S. selected three F5 EMS mutants; M.C.K., M.P., and T.W. performed bioinformatics analyses; J.S.M. and H.Z. contributed with bioinformatic and experimental experiences and assistance; P.K. and C.Z. contributed with experimental and scientific expertise. H.Š., M.K., and J.D. performed chromosome flow sorting and preparation of chromosomal DNA; J.G. and M.C.K. performed confocal microscopy investigations; G.G. and M.C.K. performed salicylic acid investigations; W.Z. contributed to the HHPred analysis; M.C.K., T.W., S.G.K., and B.K. wrote the manuscript.

\section{Competing interests}

The authors declare no competing interests.

\section{Additional information}

Supplementary information is available for this paper at https://doi.org/10.1038/s41467020-20777-x.

Correspondence and requests for materials should be addressed to T.W., S.G.K. or B.K.

Peer review information Nature Communications thanks Kostya Kanyuka, Matthew Rouse and the other, anonymous, reviewer(s) for their contribution to the peer review of this work.

Reprints and permission information is available at http://www.nature.com/reprints

Publisher's note Springer Nature remains neutral with regard to jurisdictional claims in published maps and institutional affiliations.

\begin{abstract}
(c) (i) Open Access This article is licensed under a Creative Commons (c) Attribution 4.0 International License, which permits use, sharing, adaptation, distribution and reproduction in any medium or format, as long as you give appropriate credit to the original author(s) and the source, provide a link to the Creative Commons license, and indicate if changes were made. The images or other third party material in this article are included in the article's Creative Commons license, unless indicated otherwise in a credit line to the material. If material is not included in the article's Creative Commons license and your intended use is not permitted by statutory regulation or exceeds the permitted use, you will need to obtain permission directly from the copyright holder. To view a copy of this license, visit http://creativecommons.org/ licenses/by/4.0/.
\end{abstract}

(c) The Author(s) 2021 